STUPP-07-194,

IFIC/08-15,

FTUV-08-0305

\title{
Model building by coset space dimensional reduction scheme using ten-dimensional coset spaces
}

\author{
Toshifumi Jittoh,Masafumi Koike,Takaaki Nomura,Joe Sato,Takashi Shimo- \\ mura* \\ Saitama University, Shimo-Okubo, Sakura-ku, Saitama 355-8570, Japan; \\ Departament de Física Teòrica and IFIC, Universitat de València-CSIC, \\ E-46100 Burjassot, València, Spain*
}

\begin{abstract}
We investigate the gauge-Higgs unification models within the scheme of the coset space dimensional reduction, beginning with a gauge theory in a fourteen-dimensional spacetime where extra-dimensional space has the structure of a ten-dimensional compact coset space. We found seventeen phenomenologically acceptable models through an exhaustive search for the candidates of the coset spaces, the gauge group in fourteen dimension, and fermion representation. Of the seventeen, ten models led to $\mathrm{SO}(10)(\times \mathrm{U}(1))$ GUT-like models after dimensional reduction, three models led to $\mathrm{SU}(5) \times \mathrm{U}(1)$ GUT-like models, and four to $\mathrm{SU}(3) \times \mathrm{SU}(2) \times \mathrm{U}(1) \times \mathrm{U}(1)$ StandardModel-like models. The combinations of the coset space, the gauge group in the fourteen-dimensional spacetime, and the representation of the fermion contents of such models are listed.
\end{abstract}




\section{$\S 1$. Introduction}

The Standard Model (SM) has described the interactions of the elementary particles successfully. In this model, the Higgs scalar plays an essential role in the mechanism of spontaneous breaking of the gauge symmetry from $\mathrm{SU}(3)_{\mathrm{C}} \times \mathrm{SU}(2)_{\mathrm{L}} \times \mathrm{U}(1)_{Y}$ down to $\mathrm{SU}(3)_{\mathrm{C}} \times \mathrm{U}(1)_{\mathrm{em}}$, giving masses to the elementary particles. Nevertheless, the Higgs particle itself is still undiscovered. Not only is it the last frontier of the SM, it will also provide the key clue to the physics beyond the SM, since the SM does not address even the most fundamental nature of the Higgs particle, such as its mass and the self-coupling constants.

The gauge-Higgs unification is one of attractive approaches to the physics beyond the SM in this regard ${ }^{1-3)}$ (for recent approaches, see Refs. ${ }^{4-19)}$ ). In this approach, the Higgs sector is embraced into the gauge interactions in the spacetime with dimensions larger than four, where the extra-dimensional space is compactified to a small scale to reproduce the fourdimensional spacetime. The scalar particles originate from the extra-dimensional components of the gauge field and part of the fundamental properties of Higgs scalar is determined from the gauge interactions.

We consider this approach in the framework of coset space dimensional reduction $(\mathrm{CSDR})^{20)}$ (for recent approaces, see Refs. ${ }^{21-23)}$ ). This framework introduces a compact extra-dimensional space which has the structure of a coset of Lie groups, and identifies the gauge transformation as the translation within the extra-dimensional space. This identification determines both the gauge symmetry and the particle contents of the four-dimensional theory.

Gauge theories in six- and ten-dimensional spacetime are well investigated. No known model, however, reproduced the particle content of the SM or Grand Unified Theory (GUT). ${ }^{1), 20), 24)-31 \text { ) }}$ The difficulty arises due to the insufficient dimensionality of the vector and the spinor representations of the rotational group of the extra-dimensional space, to one of which all the scalars and fermions need to belong. One of the promising ways to overcome this difficulty is to increase the dimensionality of extra-dimensional space. The higher-dimensional models enlarge these representations and thus enriches the particle contents, which hopefully include particle contents of the SM or GUT. Another merit of such models is the increase of candidates of the coset space and thus of the gauge group.

In determining the dimensionality of the extra-dimension, we pay special attention to the chiral structure of the SM and GUT. The representation of the gauge group in higherdimension needs to be complex in general to induce a chiral four-dimensional theory. The exception is the case where the dimensionality is $4 n+2$. This choice allows to start from vector-like representations, leaving larger opportunity to search for an acceptable model. Therefore we investigate fourteen-dimensional spacetime and search for GUT, GUT-like 
model, the SM and the SM-like model. A fourteen-dimensional theory is studied in terms of a bosonic string theory in a twenty-six dimensional spacetime, ${ }^{32)}$ giving another motivation of the models in the present work.

In this paper, we search for gauge theories in fourteen-dimensional spacetime which leads to a phenomenologically acceptable model. We exhaustively determined the coset spaces and the gauge groups. The scalar contents are completely determined for each case and the fermion contents are searched. Here we consider the dimensions of fermion representations less than 1025 since even larger representations yield numerous higher dimensional representations of fermion, under the gauge group of the SM or GUTs, in the four-dimensions.

This paper is organized as follows. In Sec. 2, we give a brief review of the scheme of CSDR. In Sec. 3, we consider the candidates of the theories which lead to the phenomenologically acceptable models after the dimensional reduction. We summarize our results in Sec. 4 .

\section{$\S 2$. The scheme of coset space dimensional reduction}

In this section, we recapitulate the scheme of the coset space dimensional reduction (CSDR) and the construction of the four-dimensional theory by CSDR. ${ }^{20)}$

We begin with a gauge theory with a gauge group $G$ defined on a $D$-dimensional spacetime $M^{D}$. The spacetime $M^{D}$ is assumed to be a direct product of the four-dimensional spacetime $M^{4}$ and a compact coset space $S / R$ such that $M^{D}=M^{4} \times S / R$, where $S$ is a compact Lie group and $R$ is a Lie subgroup of $S$. The dimension of the coset space $S / R$ is thus $d \equiv D-4$, implying $\operatorname{dim} S-\operatorname{dim} R=d$. This assumption on the structure of extra-dimensional space requires the group $R$ to be embedded into the group $\mathrm{SO}(d)$, which is a subgroup of the Lorentz group $\mathrm{SO}(1, D-1)$. Let us denote the coordinates of $M^{D}$ by $X^{M}=\left(x^{\mu}, y^{\alpha}\right)$, where $x^{\mu}$ and $y^{\alpha}$ are coordinates of $M^{4}$ and $S / R$, respectively. The spacetime index $M$ runs over $\mu \in\{0,1,2,3\}$ and $\alpha \in\{4,5, \cdots, D-1\}$. We define the vielbein $e_{M}{ }^{A}$ which relates the metric of the manifold $M^{D}$ (the bulk spacetime), denoted by $g_{M N}(X)$, and that of the tangent space $T_{X} M^{D}$ (the local Lorentz frame), denoted by $h_{A B}(X)$, as $g_{M N}=e_{M}{ }^{A} e_{N}{ }^{B} h_{A B}$. Here $A=(\mu, a)$, where $a \in\{4, \cdots, D\}$, is the index for the coordinates of $T_{X} M^{D}$. We conventionally use $\mu, \nu, \lambda, \cdots$ to denote the indices for $M^{4} ; \alpha, \beta, \gamma, \cdots$ for the coset space $S / R ; a, b, c, \cdots$ for the algebra of the group $S / R ; M, N, \cdots$ for $(\mu, \alpha)$; and $A, B$ for $(\mu, a)$. We introduce a gauge field $A_{M}(x, y)=\left(A_{\mu}(x, y), A_{\alpha}(x, y)\right)$, which belongs to the adjoint

representation of the gauge group $G$, and fermions $\psi(x, y)$, which lies in a representation $F$ 
of $G$. The action $S$ of this theory is given by

$$
\begin{aligned}
S= & \int d^{D} X \sqrt{-g} \\
\times( & -\frac{1}{8} g^{M N} g^{K L} \operatorname{Tr} F_{M K}(X) F_{N L}(X) \\
& \left.+\frac{1}{2} i \bar{\psi}(X) \Gamma^{A} e_{A}{ }^{M} D_{M} \psi(X)\right),
\end{aligned}
$$

where $g=\operatorname{det} g_{M N}, F_{M N}(X)=\partial_{M} A_{N}(X)-\partial_{N} A_{M}(X)-\left[A_{M}(X), A_{N}(X)\right]$ is the field strength, $D_{M}$ is the covariant derivative on $M^{D}$, and $\Gamma^{A}$ is the generators of the $D$ dimensional Clifford algebra.

The extra-dimensional space $S / R$ admits $S$ as an isometric transformation group, and we impose on $A_{M}(X)$ and $\psi(X)$ the following symmetry under this transformation in order to carry out the dimensional reduction. ${ }^{33)-38)}$ Consider a coordinate transformation which acts trivially on $x$ and gives rise to a $S$-transformation on $y$ as

$$
(x, y) \rightarrow(x, s y)
$$

where $s \in S$. We require that this coordinate transformation Eq. (2) should be compensated by a gauge transformation. This symmetry, connecting nontrivially the coordinate and gauge transformation, requires $R$ to be embedded into $G$. The symmetry further leads to the following set of the symmetric condition on the fields:

$$
\begin{aligned}
A_{\mu}(x, y)= & g(y ; s) A_{\mu}\left(x, s^{-1} y\right) g^{-1}(y ; s), \\
A_{\alpha}(x, y)= & g(y ; s) J_{\alpha}{ }^{\beta} A_{\beta}\left(x, s^{-1} y\right) g^{-1}(y ; s) \\
& +g(y ; s) \partial_{\alpha} g^{-1}(y ; s), \\
\psi(x, y)= & f(y ; s) \Omega \psi\left(x, s^{-1} y\right),
\end{aligned}
$$

where $g(y ; s)$ and $f(y ; s)$ are gauge transformations in the adjoint representation and in the representation $F$, respectively, and $J_{\alpha}{ }^{\beta}$ and $\Omega$ are the rotation in the tangent space for the vectors and spinors, respectively. These conditions of Eq. (3) make the D-dimensional Lagrangian invariant under the $S$-transformation of Eq. (2) and therefore independent of the coordinate $y$ of $S / R$. The dimensional reduction is then carried out by integrating over the coordinate $y$ to obtain the four-dimensional Lagrangian. The four-dimensional theory consists of the gauge fields $A_{\mu}$, fermions $\psi$, and in addition the scalars $\phi_{a} \equiv e_{a}{ }^{\alpha} A_{\alpha}$. The gauge group reduces to a subgroup $H$ of the original gauge group $G$. The dimensional reduction under the symmetric condition Eq. (3) and the assumption $h^{A B}=\operatorname{diag}\left(\eta^{\mu \nu},-g^{a b}\right)$, where $\eta_{\mu \nu}=\operatorname{diag}(1,-1,-1,-1)$ and $g^{a b}=\operatorname{diag}\left(a_{1}, a_{2}, \cdots, a_{d}\right)$ with $a_{i}$ 's being positive, leads to 
the four-dimensional effective Lagrangian $L^{\text {eff }}$ given by

$$
\begin{aligned}
L^{\mathrm{eff}}= & -\frac{1}{4} F_{\mu \nu}^{t} F^{t \mu \nu}+\frac{1}{2}\left(D_{\mu} \phi_{a}\right)^{t}\left(D^{\mu} \phi^{a}\right)^{t}+V(\phi) \\
& +\frac{1}{2} i \bar{\psi} \Gamma^{\mu} D_{\mu} \psi+\frac{1}{2} i \bar{\psi} \Gamma^{a} e_{a}^{\alpha} D_{\alpha} \psi,
\end{aligned}
$$

where $t$ is the index for the generators of the gauge group $G$. It is notable that the Lagrangian Eq. (44) includes the scalar potential $V(\phi)$, which is completely determined by the group structure as

$$
\begin{aligned}
V(\phi)= & -\frac{1}{4} g^{a c} g^{b d} \\
& \times \operatorname{Tr}\left[\left(f_{a b}^{C} \phi_{C}-\left[\phi_{a}, \phi_{b}\right]\right)\left(f_{c d}^{D} \phi_{D}-\left[\phi_{c}, \phi_{d}\right]\right)\right],
\end{aligned}
$$

where $C$ and $D$ runs over the indices of the algebra of $S$, and $f_{a b}{ }^{C}$ is the structure constants of the algebra of $S$. This potential may cause the spontaneous symmetry breaking, rendering the final gauge group $K$ a subgroup of the group $H$.

The scheme of CSDR substantially constrains the four-dimensional gauge group $H$ and its representations for the particle contents as shown below. First, the gauge group of the four-dimensional theory $H$ is easily identified as

$$
H=C_{G}(R)
$$

where $C_{G}(R)$ denotes the centralizer of $R$ in $G{ }^{33)}$ Note that this implies $G \supset H \times R$ up to the U(1) factors. Secondly, the representations of $H$ for the Higgs fields are specified by the following prescription. Suppose that the adjoint representations of $R$ and $G$ are decomposed according to the embeddings $S \supset R$ and $G \supset H \times R$ as

$$
\begin{aligned}
\operatorname{adj} S & =\operatorname{adj} R+\sum_{s} r_{s}, \\
\operatorname{adj} G & =(\operatorname{adj} H, 1)+(1, \operatorname{adj} R)+\sum_{g}\left(h_{g}, r_{g}\right),
\end{aligned}
$$

where $r_{s} \mathrm{~s}$ and $r_{g} \mathrm{~s}$ denote representations of $R$, and $h_{g}$ s denote representations of $H$. The representation of the scalar fields are $h_{g}$ s whose corresponding $r_{g} \mathrm{~s}$ in the decomposition Eq. (8) are contained also in the set $\left\{r_{s}\right\}$. Thirdly, the representation of $H$ for the fermion fields are determined as follows. ${ }^{39)}$ Let the group $R$ be embedded into the Lorentz group $\mathrm{SO}(d)$ in such a way that the vector representation $d$ of $\mathrm{SO}(d)$ is decomposed as

$$
d=\sum_{s} r_{s},
$$


where $r_{s}$ are the representations obtained in the decomposition Eq. (9). This embedding specifies a decomposition of the spinor representation $\sigma_{d}$ of $\mathrm{SO}(d)$ into irreducible representations $\sigma_{i}$ s of $R$ as

$$
\sigma_{d}=\sum_{i} \sigma_{i}
$$

Now the representations of $H$ for the four-dimensional fermions are found by decomposing $F$ according to $G \supset H \times R$ as

$$
F=\sum_{f}\left(h_{f}, r_{f}\right)
$$

The representations of our interest are $h_{f} \mathrm{~s}$ whose corresponding $r_{f} \mathrm{~s}$ are found in $\left\{\sigma_{i}\right\}$ obtained in Eq. (10). Note that a phenomenologically acceptable model needs chiral fermions in the four dimensions as the SM does. This is possible only when the coset space $S / R$ satisfies $\operatorname{rank} S=\operatorname{rank} R$, according to the non-trivial result due to Bott. ${ }^{44)}$ The chiral fermions are then obtained most straightforwardly when we introduce a Weyl fermion in $D=2 n$ $(n=1,2, \cdots)$ dimensions and $F$ is a complex representation. ${ }^{40)-43)}$ Interestingly, they can be obtained even if $F$ is real or pseudoreal representation, provided $D=4 n+2{ }^{40) \text {, 43) }}$ The four-dimensional fermions are doubled in these cases, and these extra fermions are eliminated by imposing the Majorana condition on the Weyl fermions in $D=4 n+2$ dimensions. ${ }^{40)}$,43) From this condition we get chiral fermions for $D=8 n+2(8 n+6)$ when $F$ is real (pseudoreal). It is therefore interesting to consider $D=6,10,14,18, \cdots$.

\section{$\S 3 . \quad$ The Search for acceptable candidates}

In this section, we search for candidates of the coset space $S / R$, the gauge group $G$, and its representation $F$ for fermions in the spacetime of the dimensionality $D=14$ for phenomenologically acceptable models based on CSDR scheme. Such models should induce a four-dimensional theory that has a gauge group $H \supset \mathrm{SU}(3) \times \mathrm{SU}(2) \times \mathrm{U}(1)$, and accomodates chiral fermions contained in the SM. This requirement constrains the $D, S / R, G, F$, and the embedding of $R$ in $G$.

Number of dimensions $D$ should be $2 n$ in order to give chiral fermions in four dimensions. We are particularly interested in the case of $D=4 n+2$, where chiral fermions can be obtained in four dimensions even if $F$ is real or pseudoreal. The simplest cases of $D=6$ and 10 are well investigated. No known model, however, reproduced the particle contents of the SM or GUT.. 1),20),24)-31) This is due to the small dimensionality of the vector and spinor representations of $\mathrm{SO}(d)$. It is difficult when $d=2$ and 6 to match $r_{s}$ s from $\mathrm{SO}(d)$ vector and $\sigma_{i} \mathrm{~s}$ from $\mathrm{SO}(d)$ spinor with $r_{g}$ s from adj $G$ and $r_{f}$ s from $F$, respectively (see Eqs. (9)-(11)). We consider a higher-dimensional spacetime to enlarge the dimensionality of $\mathrm{SO}(d)$ vector 
and spinor representations. More $r_{g} \mathrm{~s}$ and $r_{f} \mathrm{~s}$ will satisfy the matching prescription, and hence richer particle contents are obtained. Another merit of higher-dimensional spacetime is the increase of candidates of the coset space and thus of the gauge group. We thus investigate next smallest dimensionality of $D=4 n+2$, which is $D=14$.

Coset space $S / R$ of our interest should have dimension $d=D-4=10$, implying $\operatorname{dim} S-\operatorname{dim} R=10$, and should satisfy $\operatorname{rank} S=\operatorname{rank} R$ to generate chiral fermions in four dimensions. ${ }^{44)}$ These conditions limit the possible $S / R$ to the coset spaces collected in Table I. There the correspondence between the subgroup of $R$ and the subgroup of $S$ is clarified by the brackets in $R$. For example, the coset space (2) suggests direct sum of $\mathrm{SO}(7) / \mathrm{SO}(6)$ and $\mathrm{Sp}(4) /[\mathrm{SU}(2) \times \mathrm{SU}(2)]$. The factor of $R$ with subscript "max" indicates that this factor is a maximal regular subalgebra of $S$. For example, the coset (20) in Table indicates that $[\mathrm{SU}(2) \times \mathrm{U}(1)]_{\max }$ is the maximal regular subgroup of $\mathrm{Sp}(4)$. We show the embedding of $R$ in $\mathrm{SO}(10)$ in Table II]. The representations of $r_{s}$ in Eq. (9) and $\sigma_{i}$ in Eq. (10) are listed in the columns of "Branches of 10" and "Branches of 16", respectively.

The representation $F$ of $G$ for the fermions should be either complex or pseudoreal but not real, since the fermions of real representation do not allow the Majorana condition when $D=14$ and induces doubled fermion contents after the dimensional reduction. ${ }^{40), 43)}$ Table III lists the candidate groups $G$ and their complex and pseudoreal representations. Here we consider the dimensions of fermion representations less than 1025 since even larger representations yield numerous higher dimensional representations of fermion, under the gauge group of the SM or GUTs, in the four-dimensions. The representations in this table are the candidates of $F$.

We constrain the gauge group $G$ by the following two criteria once we choose $S / R$ out of the coset spaces listed in Table I. First, $G$ should have an embedding of $R$ whose centralizer $C_{G}(R)$ is appropriate as a candidate of the four-dimensional gauge group $H$ (recall Eq. (6)). In this paper, we consider the following groups as candidates of $H$ : the GUT gauge groups such as $\mathrm{E}_{6}, \mathrm{SO}(10)$, and $\mathrm{SU}(5)$; the $\mathrm{SM}$ gauge group $\mathrm{SU}(3) \times \mathrm{SU}(2) \times \mathrm{U}(1)$; and those with an extra U(1). Secondly, we consider only the regular subgroup of $G$ when we decompose it to embed $R$. We then find that no candidate of $G$ and $S / R$ that satisfy this requirement gives $\mathrm{E}_{6}, \mathrm{E}_{6} \times \mathrm{U}(1)$, and $\mathrm{SU}(5)$ as $H$. We notice that the number of $\mathrm{U}(1)$ 's in $R$ must be no more than that in $H$, since the $\mathrm{U}(1)$ 's in $R$ is also a part of its centralizer, i.e. a part of $H$. We can thus exclude (26) - (35) in Table I The candidates of $G$ for each $S / R$ satisfying the above conditions are summarized in Table IV.

Careful consideration is necessary when there are more than one branch in decomposing $G$ to its regular subgroup $H \times R$, since the different decomposition branches lead to different representations of $H$ and $R$. Two cases deserve close attention. The first is the decomposition 
Table I. A complete list of ten-dimensional coset spaces $S / R$ with $\operatorname{rank} S=\operatorname{rank} R$. The brackets in $R$ clarifies the correspondence between the subgroup of $R$ and the subgroup of $S$. The factor of $R$ with subscript "max" indicates that this factor is a maximal regular subalgebra of $S$.

\begin{tabular}{|c|c|}
\hline No. & $S / R$ \\
\hline$(1)$ & $\mathrm{SO}(11) / \mathrm{SO}(10)$ \\
\hline$(2)$ & $\mathrm{SO}(7) \times \mathrm{Sp}(4) / \mathrm{SO}(6) \times[\mathrm{SU}(2) \times \mathrm{SU}(2)]$ \\
\hline$(3)$ & $\mathrm{G}_{2} \times \mathrm{Sp}(4) / \mathrm{SU}(3) \times[\mathrm{SU}(2) \times \mathrm{SU}(2)]$ \\
\hline$(4)$ & $\mathrm{SU}(6) / \mathrm{SU}(5) \times \mathrm{U}(1)$ \\
\hline$(5)$ & $\mathrm{SO}(9) \times \mathrm{SU}(2) / \mathrm{SO}(8) \times \mathrm{U}(1)$ \\
\hline$(6)$ & $\mathrm{SO}(7) \times \mathrm{SU}(3) / \mathrm{SO}(6) \times[\mathrm{SU}(2) \times \mathrm{U}(1)]$ \\
\hline$(7)$ & $\mathrm{SU}(4) \times \mathrm{Sp}(4) /[\mathrm{SU}(3) \times \mathrm{U}(1)] \times[\mathrm{SU}(2) \times \mathrm{SU}(2)]$ \\
\hline$(8)$ & $(\mathrm{Sp}(4))^{2} \times \mathrm{SU}(2) /[\mathrm{SU}(2) \times \mathrm{SU}(2)]^{2} \times \mathrm{U}(1)$ \\
\hline$(9)$ & $\mathrm{G}_{2} \times \mathrm{SU}(3) / \mathrm{SU}(3) \times[\mathrm{SU}(2) \times \mathrm{U}(1)]$ \\
\hline$(10)$ & $\mathrm{Sp}(4) \times \mathrm{Sp}(4) /[\mathrm{SU}(2) \times \mathrm{U}(1)]_{\max } \times[\mathrm{SU}(2) \times \mathrm{SU}(2)]$ \\
\hline$(11)$ & $\mathrm{Sp}(4) \times \mathrm{Sp}(4) /[\mathrm{SU}(2) \times \mathrm{U}(1)]_{\text {non-max }} \times[\mathrm{SU}(2) \times \mathrm{SU}(2)]$ \\
\hline$(12)$ & $\mathrm{Sp}(6) \times \mathrm{SU}(2) /[\mathrm{Sp}(4) \times \mathrm{SU}(2)] \times \mathrm{U}(1)$ \\
\hline$(13)$ & $\mathrm{G}_{2} \times \mathrm{SU}(2) / \mathrm{SU}(2) \times \mathrm{SU}(2) \times \mathrm{U}(1)$ \\
\hline$(14)$ & $\mathrm{Sp}(6) / \mathrm{Sp}(4) \times \mathrm{U}(1)$ \\
\hline$(15)$ & $\mathrm{G}_{2} / \mathrm{SU}(2) \times \mathrm{U}(1)$ \\
\hline$(16)$ & $\mathrm{Sp}(4) \times \mathrm{SU}(3) \times \mathrm{SU}(2) /[\mathrm{SU}(2) \times \mathrm{SU}(2)] \times[\mathrm{SU}(2) \times \mathrm{U}(1)] \times \mathrm{U}(1)$ \\
\hline$(17)$ & $\mathrm{SU}(4) \times \mathrm{SU}(3) /[\mathrm{SU}(3) \times \mathrm{U}(1)] \times[\mathrm{SU}(2) \times \mathrm{U}(1)]$ \\
\hline$(18)$ & $\mathrm{SO}(7) \times(\mathrm{SU}(2))^{2} / \mathrm{SO}(6) \times(\mathrm{U}(1))^{2}$ \\
\hline$(19)$ & $\mathrm{SU}(5) \times \mathrm{SU}(2) /[\mathrm{SU}(4) \times \mathrm{U}(1)] \times \mathrm{U}(1)$ \\
\hline$(20)$ & $\mathrm{Sp}(4) \times \mathrm{SU}(3) /[\mathrm{SU}(2) \times \mathrm{U}(1)]_{\max } \times[\mathrm{SU}(2) \times \mathrm{U}(1)]$ \\
\hline$(21)$ & $\mathrm{Sp}(4) \times \mathrm{SU}(3) /[\mathrm{SU}(2) \times \mathrm{U}(1)]_{\text {non-max }} \times[\mathrm{SU}(2) \times \mathrm{U}(1)]$ \\
\hline$(22)$ & $\mathrm{SU}(3) \times \mathrm{Sp}(4) /[\mathrm{U}(1) \times \mathrm{U}(1)] \times[\mathrm{SU}(2) \times \mathrm{SU}(2)]$ \\
\hline$(23)$ & $\mathrm{SU}(4) \times \mathrm{SU}(2) / \mathrm{SU}(2) \times \mathrm{SU}(2) \times \mathrm{U}(1) \times \mathrm{U}(1)$ \\
\hline$(24)$ & $\mathrm{G}_{2} \times(\mathrm{SU}(2))^{2} / \mathrm{SU}(3) \times(\mathrm{U}(1))^{2}$ \\
\hline$(25)$ & $\mathrm{SU}(4) / \mathrm{SU}(2) \times \mathrm{U}(1) \times \mathrm{U}(1)$ \\
\hline$(26)$ & $\mathrm{Sp}(4) \times(\mathrm{SU}(2))^{3} /[\mathrm{SU}(2) \times \mathrm{SU}(2)] \times(\mathrm{U}(1))^{3}$ \\
\hline$(27)$ & $(\mathrm{SU}(3))^{2} \times \mathrm{SU}(2) /[\mathrm{SU}(2) \times \mathrm{U}(1)]^{2} \times \mathrm{U}(1)$ \\
\hline$(28)$ & $\mathrm{SU}(4) \times(\mathrm{SU}(2))^{2} /[\mathrm{SU}(3) \times \mathrm{U}(1)] \times(\mathrm{U}(1))^{2}$ \\
\hline$(29)$ & $\mathrm{Sp}(4) \times(\mathrm{SU}(2))^{2} /[\mathrm{SU}(2) \times \mathrm{U}(1)]_{\max } \times(\mathrm{U}(1))^{2}$ \\
\hline$(30)$ & $\mathrm{Sp}(4) \times(\mathrm{SU}(2))^{2} /[\mathrm{SU}(2) \times \mathrm{U}(1)]_{\text {non-max }} \times(\mathrm{U}(1))^{2}$ \\
\hline$(31)$ & $\mathrm{SU}(3) \times \mathrm{SU}(3) /[\mathrm{U}(1) \times \mathrm{U}(1)] \times[\mathrm{SU}(2) \times \mathrm{U}(1)]$ \\
\hline$(32)$ & $\mathrm{Sp}(4) \times \mathrm{SU}(2) /[\mathrm{U}(1) \times \mathrm{U}(1)] \times \mathrm{U}(1)$ \\
\hline$(33)$ & $\mathrm{SU}(3) \times(\mathrm{SU}(2))^{3} /[\mathrm{SU}(2) \times \mathrm{U}(1)] \times(\mathrm{U}(1))^{3}$ \\
\hline$(34)$ & $(\mathrm{SU}(2) / \mathrm{U}(1))^{5}$ \\
\hline$(35)$ & $\mathrm{SU}(3) \times(\mathrm{SU}(2))^{2} /[\mathrm{U}(1) \times \mathrm{U}(1)] \times(\mathrm{U}(1))^{2}$ \\
\hline
\end{tabular}


Table II. The decompositions of the vector representation $\mathbf{1 0}$ and the spinor representation $\mathbf{1 6}$ of $\mathrm{SO}(10)$ under $R$ 's which are listed in Table Iand have two or less U(1) factors. The representations of $r_{s}$ in Eq. (9) and $\sigma_{i}$ in Eq. (10) are listed in the columns of "Branches of 10" and "Branches of 16", respectively. The U(1) charges for the cosets (16) - (35) have a freedom of retaking the linear combination.

\begin{tabular}{|c|c|c|c|}
\hline$S / R$ & & Branches of $\mathbf{1 0}$ & Branches of $\mathbf{1 6}$ \\
\hline (1) & $\mathrm{SO}(10)$ & 10 & 16 \\
\hline$(2)$ & $(\mathrm{SO}(6), \mathrm{SU}(2), \mathrm{SU}(2))$ & $(6,1,1),(1,2,2)$ & $(4,2,1),(\overline{4}, 1,2)$ \\
\hline$(3)$ & $(\mathrm{SU}(3), \mathrm{SU}(2), \mathrm{SU}(2))$ & $(\mathbf{3}, \mathbf{1}, \mathbf{1}),(\overline{\mathbf{3}}, \mathbf{1}, \mathbf{1}),(\mathbf{1}, \mathbf{2}, \mathbf{2})$ & $(\mathbf{3}, \mathbf{2}, \mathbf{1}),(\overline{3}, \mathbf{1}, \mathbf{2}),(\mathbf{1}, \mathbf{2}, \mathbf{1}),(\mathbf{1}, \mathbf{1}, \mathbf{2})$ \\
\hline$(4)$ & $\mathrm{SU}(5)(\mathrm{U}(1))$ & $\mathbf{5}(6), \overline{\mathbf{5}}(-6)$ & $\mathbf{1}(-15), \overline{\mathbf{5}}(9), \mathbf{1 0}(-3)$ \\
\hline$(5)$ & $\mathrm{SO}(8)(\mathrm{U}(1))$ & $\mathbf{8}_{\mathrm{v}}(0), \mathbf{1}(2), \mathbf{1}(-2)$ & $\mathbf{8}_{\mathrm{s}}(-1), \mathbf{8}_{\mathrm{c}}(1)$ \\
\hline (6) & $(\mathrm{SO}(6), \mathrm{SU}(2))(\mathrm{U}(1))$ & $(\mathbf{6}, \mathbf{1})(0),(\mathbf{1}, \mathbf{2})(3),(\mathbf{1}, \mathbf{2})(-3)$ & $(\mathbf{4}, \mathbf{2})(0),(\overline{\mathbf{4}}, \mathbf{1})(3),(\overline{\mathbf{4}}, \mathbf{1})(-3)$ \\
\hline (7) & $(\mathrm{SU}(3), \mathrm{SU}(2), \mathrm{SU}(2))(\mathrm{U}(1))$ & $\begin{array}{l}(\mathbf{3}, \mathbf{1}, \mathbf{1})(-4),(\overline{\mathbf{3}}, \mathbf{1}, \mathbf{1})(4) \\
(\mathbf{1}, \mathbf{2}, \mathbf{2})(0)\end{array}$ & $\begin{array}{l}(\mathbf{3}, \mathbf{1}, \mathbf{2})(2),(\overline{\mathbf{3}}, \mathbf{2}, \mathbf{1})(-2), \\
(\mathbf{1}, \mathbf{1}, \mathbf{2})(-6),(\mathbf{1}, \mathbf{2}, \mathbf{1})(6)\end{array}$ \\
\hline$(8)$ & $(\mathrm{SU}(2), \mathrm{SU}(2), \mathrm{SU}(2), \mathrm{SU}(2))(\mathrm{U}(1))$ & $\begin{array}{l}(\mathbf{2}, \mathbf{2}, \mathbf{1}, \mathbf{1})(0),(\mathbf{1}, \mathbf{1}, \mathbf{2}, \mathbf{2})(0) \\
(\mathbf{1}, \mathbf{1}, \mathbf{1}, \mathbf{1})(2),(\mathbf{1}, \mathbf{1}, \mathbf{1}, \mathbf{1})(-2)\end{array}$ & $\begin{array}{l}(\mathbf{2}, \mathbf{1}, \mathbf{1}, \mathbf{2})(1),(\mathbf{1}, \mathbf{2}, \mathbf{1}, \mathbf{2})(-1), \\
(\mathbf{2}, \mathbf{1}, \mathbf{2}, \mathbf{1})(-1),(\mathbf{1}, \mathbf{2}, \mathbf{2}, \mathbf{1})(1)\end{array}$ \\
\hline (9) & $(\mathrm{SU}(3), \mathrm{SU}(2))(\mathrm{U}(1))$ & $\begin{array}{l}(\mathbf{3}, \mathbf{1})(0),(\overline{\mathbf{3}}, \mathbf{1})(0),(\mathbf{1}, \mathbf{2})(3) \\
(\mathbf{1}, \mathbf{2})(-3)\end{array}$ & $\begin{array}{l}(\mathbf{3}, \mathbf{2})(0),(\overline{\mathbf{3}}, \mathbf{1})(3),(\overline{\mathbf{3}}, \mathbf{1})(-3), \\
(\mathbf{1}, \mathbf{2})(0),(\mathbf{1}, \mathbf{1})(3),(\mathbf{1}, \mathbf{1})(-3)\end{array}$ \\
\hline$(10)$ & $(\mathrm{SU}(2), \mathrm{SU}(2), \mathrm{SU}(2))(\mathrm{U}(1))$ & $\begin{array}{l}(\mathbf{2}, \mathbf{2}, \mathbf{1})(0),(\mathbf{1}, \mathbf{1}, \mathbf{3})(2) \\
(\mathbf{1}, \mathbf{1}, \mathbf{3})(-2)\end{array}$ & $\begin{array}{l}(\mathbf{2}, \mathbf{1}, \mathbf{3})(-1),(\mathbf{1}, \mathbf{2}, \mathbf{3})(1) \\
(\mathbf{1}, \mathbf{2}, \mathbf{1})(3),(\mathbf{2}, \mathbf{1}, \mathbf{1})(-3)\end{array}$ \\
\hline$(11)$ & $(\mathrm{SU}(2), \mathrm{SU}(2), \mathrm{SU}(2))(\mathrm{U}(1))$ & $\begin{array}{l}(\mathbf{2}, \mathbf{2}, \mathbf{1})(0),(\mathbf{1}, \mathbf{1}, \mathbf{2})(1) \\
(\mathbf{1}, \mathbf{1}, \mathbf{2})(-1),(\mathbf{1}, \mathbf{1}, \mathbf{1})(2) \\
(\mathbf{1}, \mathbf{1}, \mathbf{1})(-2)\end{array}$ & $\begin{array}{l}(\mathbf{1}, \mathbf{2}, \mathbf{2})(-1),(\mathbf{1}, \mathbf{2}, \mathbf{1})(0) \\
(\mathbf{1}, \mathbf{2}, \mathbf{1})(2),(\mathbf{2}, \mathbf{1}, \mathbf{2})(1) \\
(\mathbf{2}, \mathbf{1}, \mathbf{1})(0),(\mathbf{2}, \mathbf{1}, \mathbf{1})(-2)\end{array}$ \\
\hline$(12)$ & $(\mathrm{Sp}(4), \mathrm{SU}(2))(\mathrm{U}(1))$ & $(\mathbf{4}, \mathbf{2})(0),(\mathbf{1}, \mathbf{1})(2),(\mathbf{1}, \mathbf{1})(-2)$ & $(\mathbf{5}, \mathbf{1})(-1),(\mathbf{1}, \mathbf{3})(-1),(\mathbf{4}, \mathbf{2})(1)$ \\
\hline (13) & $(\mathrm{SU}(2), \mathrm{SU}(2))(\mathrm{U}(1))$ & $(\mathbf{4}, \mathbf{2})(0),(\mathbf{1}, \mathbf{1})(2),(\mathbf{1}, \mathbf{1})(-2)$ & $(\mathbf{4}, \mathbf{2})(1),(\mathbf{5}, \mathbf{1})(-1),(\mathbf{1}, \mathbf{3})(-1)$ \\
\hline$(14)$ & $\mathrm{Sp}(4)(\mathrm{U}(1))$ & $\mathbf{4}(1), \mathbf{4}(-1), \mathbf{1}(2), \mathbf{1}(-2)$ & $\mathbf{5}(1), \mathbf{4}(-2), \mathbf{4}(0), \mathbf{1}(3), \mathbf{1}(1), \mathbf{1}(-1)$ \\
\hline$(15 a)$ & $\mathrm{SU}(2)(\mathrm{U}(1))$ & $\begin{array}{l}\mathbf{2}(3), \mathbf{2}(-3), \mathbf{2}(1), \mathbf{2}(-1), \\
\mathbf{1}(-2), \mathbf{1}(2)\end{array}$ & $\begin{array}{l}\mathbf{3}(1), \mathbf{2}(-4), \mathbf{2}(2), \mathbf{2}(-2), \\
\mathbf{2}(0), \mathbf{1}(5), \mathbf{1}(3), \mathbf{1}(-3), \mathbf{1}(1), \mathbf{1}(-1)\end{array}$ \\
\hline$(15 \mathrm{~b})$ & $\mathrm{SU}(2)(\mathrm{U}(1))$ & $\mathbf{4}(1), \mathbf{4}(-1), \mathbf{1}(2), \mathbf{1}(-2)$ & $\mathbf{5}(1), \mathbf{4}(-2), \mathbf{4}(0), \mathbf{1}(3), \mathbf{1}(1), \mathbf{1}(-1)$, \\
\hline$(16)$ & $(\mathrm{SU}(2), \mathrm{SU}(2), \mathrm{SU}(2))(\mathrm{U}(1), \mathrm{U}(1))$ & $\begin{array}{l}(\mathbf{2}, \mathbf{2}, \mathbf{1})(0,0),(\mathbf{1}, \mathbf{1}, \mathbf{2})(3,0) \\
(\mathbf{1}, \mathbf{1}, \mathbf{2})(-3,0),(\mathbf{1}, \mathbf{1}, \mathbf{1})(0,2) \\
(\mathbf{1}, \mathbf{1}, \mathbf{1})(0,-2)\end{array}$ & $\begin{array}{l}(\mathbf{2}, \mathbf{1}, \mathbf{2})(0,1),(\mathbf{1}, \mathbf{2}, \mathbf{2})(0,-1) \\
(\mathbf{2}, \mathbf{1}, \mathbf{1})(3,-1),(\mathbf{2}, \mathbf{1}, \mathbf{1})(-3,-1) \\
(\mathbf{1}, \mathbf{2}, \mathbf{1})(3,1),(\mathbf{1}, \mathbf{2}, \mathbf{1})(-3,1)\end{array}$ \\
\hline$(17)$ & $(\mathrm{SU}(3), \mathrm{SU}(2))(\mathrm{U}(1), \mathrm{U}(1))$ & $\begin{array}{l}(\mathbf{3}, \mathbf{1})(0,-4),(\overline{\mathbf{3}}, \mathbf{1})(0,4), \\
(\mathbf{1}, \mathbf{2})(3,0),(\mathbf{1}, \mathbf{2})(-3,0)\end{array}$ & $\begin{array}{l}(\mathbf{3}, \mathbf{2})(0,2),(\overline{\mathbf{3}}, \mathbf{1})(3,-2),(\overline{\mathbf{3}}, \mathbf{1})(-3,-2), \\
(\mathbf{1}, \mathbf{2})(0,-6),(\mathbf{1}, \mathbf{1})(3,6),(\mathbf{1}, \mathbf{1})(-3,6)\end{array}$ \\
\hline (18) & $\mathrm{SO}(6)(\mathrm{U}(1), \mathrm{U}(1))$ & $\begin{array}{l}\mathbf{6}(0,0), \mathbf{1}(2,0), \mathbf{1}(-2,0) \\
\mathbf{1}(0,2), \mathbf{1}(0,-2)\end{array}$ & $\begin{array}{l}\mathbf{4}(1,-1), \mathbf{4}(-1,1), \overline{\mathbf{4}}(1,1) \\
\overline{\mathbf{4}}(-1,-1)\end{array}$ \\
\hline (19) & $\mathrm{SU}(4)(\mathrm{U}(1), \mathrm{U}(1))$ & $\begin{array}{l}\mathbf{4}(0,-5), \overline{\mathbf{4}}(0,5), \mathbf{1}(2,0) \\
\mathbf{1}(-2,0)\end{array}$ & $\begin{array}{l}\mathbf{6}(-1,0), \mathbf{4}(1,5), \overline{\mathbf{4}}(1,-5), \\
\mathbf{1}(-1,10), \mathbf{1}(-1,-10)\end{array}$ \\
\hline$(20)$ & $(\mathrm{SU}(2), \mathrm{SU}(2))(\mathrm{U}(1), \mathrm{U}(1))$ & $\begin{array}{l}(\mathbf{3}, \mathbf{1})(0,2),(\mathbf{3}, \mathbf{1})(0,-2) \\
(\mathbf{1}, \mathbf{2})(3,0),(\mathbf{1}, \mathbf{2})(-3,0)\end{array}$ & $\begin{array}{l}(\mathbf{3}, \mathbf{2})(0,-1),(\mathbf{3}, \mathbf{1})(3,1),(\mathbf{3}, \mathbf{1})(-3,1) \\
(\mathbf{1}, \mathbf{2})(0,3),(\mathbf{1}, \mathbf{1})(3,-3),(\mathbf{1}, \mathbf{1})(-3,-3)\end{array}$ \\
\hline$(21)$ & $(\mathrm{SU}(2), \mathrm{SU}(2))(\mathrm{U}(1), \mathrm{U}(1))$ & $\begin{array}{l}(\mathbf{2}, \mathbf{1})(1,0),(\mathbf{2}, \mathbf{1})(-1,0) \\
(\mathbf{1}, \mathbf{2})(0,3),(\mathbf{1}, \mathbf{2})(0,-3) \\
(\mathbf{1}, \mathbf{1})(2,0),(\mathbf{1}, \mathbf{1})(-2,0)\end{array}$ & $\begin{array}{l}(\mathbf{2}, \mathbf{2})(-1,0),(\mathbf{1}, \mathbf{2})(2,0),(\mathbf{1}, \mathbf{2})(0,0), \\
(\mathbf{2}, \mathbf{1})(1,3),(\mathbf{2}, \mathbf{1})(1,-3),(\mathbf{1}, \mathbf{1})(0,3), \\
(\mathbf{1}, \mathbf{1})(0,-3),(\mathbf{1}, \mathbf{1})(-2,3),(\mathbf{1}, \mathbf{1})(-2,-3),\end{array}$ \\
\hline$(22)$ & $(\mathrm{SU}(2), \mathrm{SU}(2))(\mathrm{U}(1), \mathrm{U}(1))$ & $\begin{array}{l}(\mathbf{2}, \mathbf{2})(0,0),(\mathbf{1}, \mathbf{1})(a, c), \\
(\mathbf{1}, \mathbf{1})(b, d),(\mathbf{1}, \mathbf{1})(-a,-c) \\
(\mathbf{1}, \mathbf{1})(-b,-d) \\
(\mathbf{1}, \mathbf{1})(a+b, c+d), \\
(\mathbf{1}, \mathbf{1})(-a-b,-c-d)\end{array}$ & $\begin{array}{l}(\mathbf{2}, \mathbf{1})(0,0),(\mathbf{1}, \mathbf{2})(0,0) \\
(\mathbf{2}, \mathbf{1})(b, d),(\mathbf{2}, \mathbf{1})(a, c) \\
(\mathbf{2}, \mathbf{1})(-a-b,-c-d) \\
(\mathbf{1}, \mathbf{2})(a+b, c+d) \\
(\mathbf{1}, \mathbf{2})(-a,-c),(\mathbf{1}, \mathbf{2})(-b,-d)\end{array}$ \\
\hline
\end{tabular}


Table II. (Continued.)

\begin{tabular}{|c|c|c|c|}
\hline$S / R$ & & Branches of $\mathbf{1 0}$ & Branches of $\mathbf{1 6}$ \\
\hline$(23)$ & $(\mathrm{SU}(2), \mathrm{SU}(2))(\mathrm{U}(1), \mathrm{U}(1))$ & $\begin{array}{l}(\mathbf{2}, \mathbf{2})(0,2),(\mathbf{2}, \mathbf{2})(0,-2) \\
(\mathbf{1}, \mathbf{1})(2,0),(\mathbf{1}, \mathbf{1})(-2,0)\end{array}$ & $\begin{array}{l}(\mathbf{3}, \mathbf{1})(-1,0),(\mathbf{1}, \mathbf{3})(-1,0),(\mathbf{2}, \mathbf{2})(1,-2), \\
(\mathbf{2}, \mathbf{2})(1,2),(\mathbf{1}, \mathbf{1})(-1,4),(\mathbf{1}, \mathbf{1})(-1,-4),\end{array}$ \\
\hline$(24)$ & $\mathrm{SU}(3)(\mathrm{U}(1), \mathrm{U}(1))$ & $\begin{array}{l}\mathbf{3}(0,0), \overline{\mathbf{3}}(0,0), \mathbf{1}(2,0) \\
\mathbf{1}(-2,0), \mathbf{1}(0,2), \mathbf{1}(0,-2)\end{array}$ & $\begin{array}{l}\mathbf{3}(1,-1), \mathbf{3}(-1,1), \overline{\mathbf{3}}(1,1), \overline{\mathbf{3}}(-1,-1), \\
\mathbf{1}(1,-1), \mathbf{1}(-1,1), \mathbf{1}(1,1), \mathbf{1}(-1,-1)\end{array}$ \\
\hline$(25)$ & $\mathrm{SU}(2)(\mathrm{U}(1), \mathrm{U}(1))$ & $\begin{array}{l}\mathbf{2}(-1,2), \mathbf{2}(1,2), \mathbf{2}(-1,-2) \\
\mathbf{2}(1,-2), \mathbf{1}(2,0), \mathbf{1}(-2,0)\end{array}$ & $\begin{array}{l}\mathbf{3}(-1,0), \mathbf{2}(2,2), \mathbf{2}(0,2), \mathbf{2}(0,-2), \mathbf{2}(2,-2), \\
\mathbf{1}(-1,4), \mathbf{1}(-1,-4), \mathbf{1}(-3,0), \mathbf{1}(1,0), \mathbf{1}(-1,0)\end{array}$ \\
\hline$(26)$ & $(\mathrm{SU}(2), \mathrm{SU}(2))(\mathrm{U}(1), \mathrm{U}(1), \mathrm{U}(1))$ & $\begin{array}{l}(\mathbf{2}, \mathbf{2})(0,0,0),(\mathbf{1}, \mathbf{1})(2,0,0), \\
(\mathbf{1}, \mathbf{1})(-2,0,0),(\mathbf{1}, \mathbf{1})(0,2,0), \\
(\mathbf{1}, \mathbf{1})(0,-2,0),(\mathbf{1}, \mathbf{1})(0,0,2), \\
(\mathbf{1}, \mathbf{1})(0,0,-2)\end{array}$ & $\begin{array}{l}(\mathbf{2}, \mathbf{1})(1,1,1),(\mathbf{2}, \mathbf{1})(-1,-1,1) \\
(\mathbf{2}, \mathbf{1})(1,-1,-1),(\mathbf{2}, \mathbf{1})(-1,1,-1), \\
(\mathbf{1}, \mathbf{2})(1,-1,1),(\mathbf{1}, \mathbf{2})(-1,1,1) \\
(\mathbf{1}, \mathbf{2})(1,1,-1),(\mathbf{1}, \mathbf{2})(-1,-1,-1)\end{array}$ \\
\hline$(27)$ & $(\mathrm{SU}(2), \mathrm{SU}(2))(\mathrm{U}(1), \mathrm{U}(1), \mathrm{U}(1))$ & $\begin{array}{l}(\mathbf{2}, \mathbf{1})(3,0,0),(\mathbf{2}, \mathbf{1})(-3,0,0) \\
(\mathbf{1}, \mathbf{2})(0,3,0),(\mathbf{1}, \mathbf{2})(0,-3,0) \\
(\mathbf{1}, \mathbf{1})(0,0,2),(\mathbf{1}, \mathbf{1})(0,0,-2)\end{array}$ & $\begin{array}{l}(\mathbf{2}, \mathbf{2})(0,0,-1),(\mathbf{2}, \mathbf{1})(0,3,1), \\
(\mathbf{2}, \mathbf{1})(0,-3,1),(\mathbf{1}, \mathbf{2})(3,0,1), \\
(\mathbf{1}, \mathbf{2})(-3,0,1),(\mathbf{1}, \mathbf{1})(3,3,-1), \\
(\mathbf{1}, \mathbf{1})(-3,3,-1),(\mathbf{1}, \mathbf{1})(3,-3,-1), \\
(\mathbf{1}, \mathbf{1})(-3,-3,-1)\end{array}$ \\
\hline$(28)$ & $\mathrm{SU}(3)(\mathrm{U}(1), \mathrm{U}(1), \mathrm{U}(1))$ & $\begin{array}{l}\mathbf{3}(-4,0,0), \overline{\mathbf{3}}(4,0,0), \\
\mathbf{1}(0,2,0), \mathbf{1}(0,-2,0), \\
\mathbf{1}(0,0,2), \mathbf{1}(0,0,-2)\end{array}$ & $\begin{array}{l}\mathbf{3}(2,-1,1), \mathbf{3}(2,1,-1), \\
\overline{\mathbf{3}}(-2,1,1), \overline{\mathbf{3}}(-2,-1,-1), \\
\mathbf{1}(6,1,1), \mathbf{1}(-6,-1,1), \\
\mathbf{1}(-6,1,-1), \mathbf{1}(6,-1,-1)\end{array}$ \\
\hline$(29)$ & $\mathrm{SU}(2)(\mathrm{U}(1), \mathrm{U}(1), \mathrm{U}(1))$ & $\begin{array}{l}\mathbf{3}(2,0,0), \mathbf{3}(-2,0,0), \\
\mathbf{1}(0,2,0), \mathbf{1}(0,-2,0), \\
\mathbf{1}(0,0,2), \mathbf{1}(0,0,-2)\end{array}$ & $\begin{array}{l}\mathbf{3}(-1,1,1), \mathbf{3}(-1,-1,-1), \\
\mathbf{3}(1,1,-1), \mathbf{3}(1,-1,1) \\
\mathbf{1}(3,1,1), \mathbf{1}(3,-1,-1) \\
\mathbf{1}(-3,1,-1), \mathbf{1}(-3,-1,1)\end{array}$ \\
\hline$(30)$ & $\mathrm{SU}(2)(\mathrm{U}(1), \mathrm{U}(1), \mathrm{U}(1))$ & $\begin{array}{l}\mathbf{2}(1,0,0), \mathbf{2}(-1,0,0), \\
\mathbf{1}(2,0,0), \mathbf{1}(-2,0,0), \\
\mathbf{1}(0,2,0), \mathbf{1}(0,-2,0), \\
\mathbf{1}(0,0,2), \mathbf{1}(0,0,-2)\end{array}$ & $\begin{array}{l}\mathbf{2}(1,1,-1), \mathbf{2}(1,-1,1), \\
\mathbf{2}(-1,1,1), \mathbf{2}(-1,-1,-1), \\
\mathbf{1}(2,1,1) \mathbf{1}(2,-1,-1), \\
\mathbf{1}(-2,1,-1) \mathbf{1}(-2,-1,1), \\
\mathbf{1}(0,1,1) \mathbf{1}(0,-1,-1), \\
\mathbf{1}(0,1,-1), \mathbf{1}(0,-1,1)\end{array}$ \\
\hline$(31)$ & $\mathrm{SU}(2)(\mathrm{U}(1), \mathrm{U}(1), \mathrm{U}(1))$ & $\begin{array}{l}\mathbf{2}(3,0,0), \mathbf{2}(-3,0,0), \\
\mathbf{1}(0,2,0), \mathbf{1}(0,-2,0), \\
\mathbf{1}(0,1,3), \mathbf{1}(0,-1,-3), \\
\mathbf{1}(0,1,-3), \mathbf{1}(0,-1,3)\end{array}$ & $\begin{array}{l}\mathbf{2}(0,1,3), \mathbf{2}(0,1,-3), \\
\mathbf{2}(0,0,0), \mathbf{2}(0,-2,0), \\
\mathbf{1}(3,2,0), \mathbf{1}(-3,2,0), \\
\mathbf{1}(3,0,0), \mathbf{1}(-3,0,0) \\
\mathbf{1}(3,-1,3), \mathbf{1}(-3,-1,3), \\
\mathbf{1}(3,-1,-3), \mathbf{1}(-3,-1,-3)\end{array}$ \\
\hline$(32)$ & $(\mathrm{U}(1), \mathrm{U}(1), \mathrm{U}(1))$ & $\begin{array}{l}(2,0,0),(-2,0,0),(0,-2,0), \\
(0,2,0),(0,0,2),(0,0,-2), \\
(2,2,0),(-2,-2,0),(2,-2,0), \\
(-2,2,0)\end{array}$ & $\begin{array}{l}(3,1,-1),(3,-1,1),(-3,-1,-1), \\
(-3,1,1),(1,3,1),(-1,-3,1) \\
(-1,3,-1),(1,-3,1),(-1,1,1), \\
(1,-1,1),(1,-1,-1),(-1,1,1) \\
(1,1,1),(-1,-1,1),(1,1,-1), \\
(-1,-1,-1)\end{array}$ \\
\hline
\end{tabular}


Table II. (Continued.)

\begin{tabular}{llll}
\hline$S / R$ & & Branches of $\mathbf{1 0}$ & Branches of $\mathbf{1 6}$ \\
\hline \hline$(33)$ & $\mathrm{SU}(2)(\mathrm{U}(1), \mathrm{U}(1), \mathrm{U}(1), \mathrm{U}(1))$ & $\mathbf{2}(3,0,0,0), \mathbf{2}(-3,0,0,0)$, & $\mathbf{2}(0,1,-1,1), \mathbf{2}(0,-1,1,1)$, \\
& $\mathbf{1}(0,2,0,0), \mathbf{1}(0,-2,0,0)$, & $\mathbf{2}(0,1,1,-1) \mathbf{2}(0,-1,-1,-1)$, \\
& $\mathbf{1}(0,0,2,0), \mathbf{1}(0,0,-2,0)$, & $\mathbf{1}(3,1,1,1), \mathbf{1}(-3,1,1,1)$, \\
& $\mathbf{1}(0,0,0,2), \mathbf{1}(0,0,0,-2)$ & $\mathbf{1}(3,-1-1,1), \mathbf{1}(-3,-1,-1,1)$, \\
& & $\mathbf{1}(3,1,-1,-1), \mathbf{1}(-3,1,-1,-1)$, \\
& & $\mathbf{1}(3,-1,1,-1), \mathbf{1}(-3,-1,1,-1)$ \\
\hline$(34) \quad(\mathrm{U}(1), \mathrm{U}(1), \mathrm{U}(1), \mathrm{U}(1), \mathrm{U}(1))$ & $(2,0,0,0,0),(-2,0,0,0,0)$, & $(1,1,1,-1,1),(-1,-1,1,-1,1)$, \\
& & $(0,2,0,0,0),(0,-2,0,0,0)$, & $(1,1,-1,1,1),(-1,-1,-1,1,1)$, \\
& $(0,0,2,0,0),(0,0,-2,0,0)$, & $(1,1,1,1,-1),(-1,-1,1,1,-1)$, \\
& $(0,0,0,2,0),(0,0,0,-2,0)$, & $(1,1,-1,-1,-1),(-1,-1,-1,-1,-1)$, \\
& $(0,0,0,0,2),(0,0,0,0,-2)$ & $(1,-1,1,1,1),,(-1,1,1,1,1)$, \\
& & $(1,-1,-1,-1,1),(-1,1,-1,-1,1)$, \\
& & $(1,-1,1,-1,-1),(-1,1,1,-1,-1)$, \\
& & $(1,-1,-1,1,-1),(-1,1,-1,1,-1)$ \\
\hline$(35)$ & $(\mathrm{U}(1), \mathrm{U}(1), \mathrm{U}(1), \mathrm{U}(1))$ & $(1,3,0,0),(-1,-3,0,0)$, & $(2,0,-1,1),(-2,0,1,1)$, \\
& $(-1,3,0,0),(1,-3,0,0)$, & $(2,0,1,-1),(-2,0,-1,-1)$, \\
& $(2,0,0,0),(-2,0,0,0)$, & $(0,0,-1,1),(0,0,1,1)$, \\
& $(0,0,2,0),(0,0,-2,0)$, & $(0,0,1,-1),(0,0,-1,-1)$, \\
& $(0,0,0,2),(0,0,0,-2)$ & $(1,3,1,1),(1,-3,1,1)$, \\
& & $(-1,3,-1,1),(-1,-3,-1,1)$, \\
& & $(1,3,-1,-1),(1,-3,-1,-1)$, \\
& & &
\end{tabular}

of $\mathrm{SO}(2 n+1)$. It has essentially two distinct branches of decomposition, one being

$$
\mathrm{SO}(2 n+1) \supset \mathrm{SO}\left(2 k_{0}+1\right) \times \prod_{i} \mathrm{SO}\left(2 k_{i}\right)
$$

and the other being

$$
\mathrm{SO}(2 n+1) \supset \mathrm{SO}(2 n) \supset \prod_{i} \mathrm{SO}\left(2 k_{i}\right)
$$

An example is the decomposition of $\mathrm{Sp}(4) \simeq \mathrm{SO}(5)$ into $\mathrm{SU}(2) \times \mathrm{U}(1)$. One of the two branches of decomposition is $\mathrm{Sp}(4) \supset \mathrm{SU}(2) \times \mathrm{U}(1)$, which is equivalent to $\mathrm{SO}(5) \supset \mathrm{SO}(3) \times$ $\mathrm{SO}(2)$, corresponding to Eq. (12). The other branch is $\mathrm{Sp}(4) \simeq \mathrm{SO}(5) \supset \mathrm{SO}(4) \simeq \mathrm{SU}(2) \times$ $\mathrm{SU}(2) \supset \mathrm{SU}(2) \times \mathrm{U}(1)$, corresponding to Eq. (13). The two branches of decomposition lead to different branching of the representations. The second is the normalization of $\mathrm{U}(1)$ charge. The different normalizations provide different representations of $H$ for four-dimensional fields.

\section{1. $H=\mathrm{SO}(10)(\times \mathrm{U}(1))$}

First we search for viable $\mathrm{SO}(10)$ models in four dimensions. We list below the combinations of $S / R, G$ and $F$ that provide $H=\mathrm{SO}(10)(\times \mathrm{U}(1))$ and the representations which 
Table III. The gauge groups that have either complex or pseudoreal representations and their complex and pseudoreal representations whose dimension is no larger than 1024. ${ }^{45)}$ The groups $\mathrm{SU}(8)$ and $\mathrm{SU}(9)$ are not listed here since they do not lead to the four-dimensional gauge group

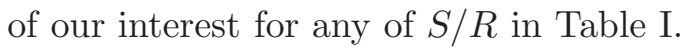

\begin{tabular}{|c|c|c|}
\hline Group & Complex representations & Pseudoreal representations \\
\hline$\overline{\mathrm{SU}(7)}$ & $\overline{221,28,35,84,112,140, \cdots}$ & \\
\hline $\mathrm{SO}(12)$ & & $32,32^{\prime}, 352,352^{\prime}$ \\
\hline $\mathrm{SO}(13)$ & & 64,768 \\
\hline $\mathrm{Sp}(12)$ & & 208,364 \\
\hline $\mathrm{E}_{6}$ & $27,351,351^{\prime}$ & \\
\hline $\mathrm{SO}(14)$ & 64,832 & \\
\hline $\mathrm{Sp}(14)$ & & $350,560,896$ \\
\hline $\operatorname{Sp}(16)$ & & 544,816 \\
\hline $\mathrm{SU}(10)$ & $45,55,120,210,220,330, \cdots$ & \\
\hline $\mathrm{SO}(18)$ & 256 & \\
\hline $\mathrm{SO}(19)$ & & 512 \\
\hline $\operatorname{Sp}(18)$ & & 798 \\
\hline $\mathrm{SO}(20)$ & & 512 \\
\hline $\mathrm{SO}(21)$ & & 1024 \\
\hline
\end{tabular}

contain field contents of the SM for the scalars and the fermions. We indicate the coset $S / R$ with its number assigned in Table 1 The embedding of $R$ into $G$ is shown for each candidates since this embedding uniquely determines all the representations of the scalars and fermions in the four-dimensional theory. In Table $\mathrm{V}$, we show all the field contents in four dimensions for each combination of $(S / R, G, F)$.

(a) $S / R(11)=\mathrm{Sp}(4) \times \mathrm{Sp}(4) /[\mathrm{SU}(2) \times \mathrm{U}(1)]_{\text {non-max }} \times[\mathrm{SU}(2) \times \mathrm{SU}(2)], G=\mathrm{SO}(19)$, and $F=512$.

We embed $R$ in the subgroup $\mathrm{SU}(2) \times \mathrm{SU}(2) \times \mathrm{SU}(2) \times \mathrm{U}(1)$ of $\mathrm{SO}(19)$ according to the decomposition

$$
\begin{aligned}
\mathrm{SO}(19) & \supset \mathrm{SO}(10) \times \mathrm{SO}(9) \\
& \supset \mathrm{SO}(10) \times \mathrm{SU}(4) \times \mathrm{SU}(2) \\
& \supset \mathrm{SO}(10) \times \mathrm{SU}(2) \times \mathrm{SU}(2) \times \mathrm{SU}(2) \times \mathrm{U}(1) .
\end{aligned}
$$


Table IV. The allowed candidates of the gauge group $G$ for each choice of $H$ and $S / R$. The top

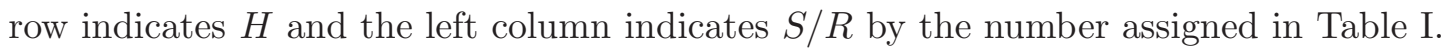

\begin{tabular}{|c|c|c|c|c|c|}
\hline & $\mathrm{SO}(10)$ & $\mathrm{SO}(10) \times \mathrm{U}(1)$ & $\mathrm{SU}(5) \times \mathrm{U}(1)$ & $\mathrm{SU}(3) \times \mathrm{SU}(2) \times \mathrm{U}(1)$ & $\mathrm{SU}(3) \times \mathrm{SU}(2) \times \mathrm{U}(1) \times \mathrm{U}(1)$ \\
\hline (1) & $\mathrm{SO}(20)$ & & & & \\
\hline (2) & $\mathrm{SO}(20)$ & & & & \\
\hline$(4)$ & & $\mathrm{SO}(20), \mathrm{SO}(21)$ & $\mathrm{SU}(10)$ & & $\mathrm{SU}(10), \mathrm{SO}(18), \mathrm{SO}(19)$ \\
\hline$(5)$ & & $\mathrm{SO}(20), \mathrm{SO}(21)$ & $\mathrm{SO}(18), \mathrm{SO}(19)$ & & $\mathrm{SO}(18), \mathrm{SO}(19)$ \\
\hline$(6)$ & & $\mathrm{SO}(20), \mathrm{SO}(21)$ & $\mathrm{SO}(19)$ & & $\mathrm{SO}(18), \mathrm{SO}(19), \mathrm{Sp}(18)$ \\
\hline$(7)$ & & & & & $\mathrm{SO}(19), \mathrm{Sp}(18)$ \\
\hline$(8)$ & & $\mathrm{SO}(20), \mathrm{SO}(21)$ & $\mathrm{SO}(18), \mathrm{SO}(19), \mathrm{Sp}(18)$ & $\mathrm{Sp}(16)$ & $\mathrm{SO}(18), \mathrm{SO}(19), \mathrm{Sp}(18)$ \\
\hline$(9)$ & & & & & $\mathrm{Sp}(16)$ \\
\hline$(10)$ & & $\mathrm{SO}(18), \mathrm{SO}(19)$ & $\mathrm{Sp}(16)$ & $\mathrm{SO}(14), \mathrm{Sp}(14)$ & $\mathrm{Sp}(16)$ \\
\hline$(11)$ & & $\mathrm{SO}(18), \mathrm{SO}(19)$ & $\mathrm{Sp}(16)$ & $\mathrm{SO}(14), \mathrm{Sp}(14)$ & $\mathrm{Sp}(16)$ \\
\hline$(12)$ & & $\mathrm{SO}(19)$ & $\mathrm{Sp}(16)$ & $\mathrm{Sp}(14)$ & $\mathrm{Sp}(16)$ \\
\hline$(13)$ & & & $\mathrm{SO}(14), \mathrm{Sp}(14)$ & $\mathrm{SO}(13), \mathrm{Sp}(12)$ & $\mathrm{SO}(14), \mathrm{Sp}(14)$ \\
\hline$(14)$ & & & $\operatorname{Sp}(14)$ & $\mathrm{Sp}(12)$ & $\mathrm{Sp}(16)$ \\
\hline$(15)$ & & $\mathrm{SO}(14)$ & $\mathrm{SU}(7), \mathrm{SO}(13), \mathrm{Sp}(12)$ & $\mathrm{SO}(10), \mathrm{SO}(11), \mathrm{Sp}(10)$ & $\mathrm{SU}(7), \mathrm{SO}(12), \mathrm{SO}(13), \mathrm{Sp}(12), \mathrm{E}_{6}$ \\
\hline$(16)$ & & & & & $\mathrm{Sp}(16)$ \\
\hline$(17)$ & & & & & $\mathrm{Sp}(16)$ \\
\hline$(18)$ & & & & & $\mathrm{SU}(9), \mathrm{Sp}(16)$ \\
\hline$(19)$ & & & & & $\mathrm{SU}(9), \mathrm{Sp}(16)$ \\
\hline$(20)$ & & & & & $\mathrm{SO}(14), \mathrm{Sp}(14)$ \\
\hline$(21)$ & & & & & $\mathrm{SO}(14), \mathrm{Sp}(14)$ \\
\hline$(22)$ & & & & & $\mathrm{SO}(14), \mathrm{Sp}(14)$ \\
\hline$(23)$ & & & & & $\mathrm{SO}(14), \mathrm{Sp}(14)$ \\
\hline$(24)$ & & & & & $\mathrm{SU}(8), \mathrm{Sp}(14)$ \\
\hline$(25)$ & & & & & $\mathrm{SU}(7), \mathrm{SO}(12), \mathrm{SO}(13), \mathrm{Sp}(12), \mathrm{E}_{6}$ \\
\hline
\end{tabular}

Table V. The field contents in four dimensions with $H=\mathrm{SO}(10)(\times \mathrm{U}(1))$ for each combination of $(S / R, G, F)$. Coset spaces are indicated by the number assigned in Table \. Numbers in a superscript of the representations denote its multiplicity.

\begin{tabular}{l|l|l||l|l|l}
\hline \multicolumn{1}{l|}{$14 \mathrm{D}$ model } & $4 \mathrm{D}$ model & \\
\hline$S / R$ & $G$ & $F$ & $H$ & Scalars & Fermions \\
\hline \hline$(1)$ & $\mathrm{SO}(20)$ & $\mathbf{5 1 2}$ & $\mathrm{SO}(10)$ & $\mathbf{1 0}$ & $\mathbf{1 6}$ \\
\hline$(2)$ & $\mathrm{SO}(20)$ & $\mathbf{5 1 2}$ & $\mathrm{SO}(10)$ & $\{\mathbf{1 0}\}^{2}$ & $\{\mathbf{1 6}\}^{2}$ \\
\hline$(4)$ & $\mathrm{SO}(20)$ & $\mathbf{5 1 2}$ & $\mathrm{SO}(10) \times \mathrm{U}(1)$ & $\mathbf{1 0}(2), \mathbf{1 0}(-2)$ & $\mathbf{1 6}(-1), \mathbf{1 6}(3), \mathbf{1 6}(-5)$ \\
\hline$(5)$ & $\mathrm{SO}(20)$ & $\mathbf{5 1 2}$ & $\mathrm{SO}(10) \times \mathrm{U}(1)$ & $\mathbf{1 0}(0), \mathbf{1 0}(2), \mathbf{1 0}(-2)$ & $\mathbf{1 6}(1), \mathbf{1 6}(-1)$ \\
\hline$(6)$ & $\mathrm{SO}(20)$ & $\mathbf{5 1 2}$ & $\mathrm{SO}(10) \times \mathrm{U}(1)$ & $\mathbf{1 0}(0), \mathbf{1 0}(1), \mathbf{1 0}(-1)$ & $\mathbf{1 6}(0), \mathbf{1 6}(1), \mathbf{1 6}(-1)$ \\
\hline$(8)$ & $\mathrm{SO}(20)$ & $\mathbf{5 1 2}$ & $\mathrm{SO}(10) \times \mathrm{U}(1)$ & $\mathbf{1 0}(0), \mathbf{1 0}(0), \mathbf{1 0}(2), \mathbf{1 0}(-2)$ & $\mathbf{1 6}(1), \mathbf{1 6}(1), \mathbf{1 6}(-1), \mathbf{1 6}(-1)$ \\
\hline$(10)$ & $\mathrm{SO}(18)$ & $\mathbf{2 5 6}$ & $\mathrm{SO}(10) \times \mathrm{U}(1)$ & $\mathbf{1 0}(0)$ & $\mathbf{1 6}(3), \mathbf{1 6}(-3), \overline{\mathbf{1 6}}(-3), \overline{\mathbf{1 6}}(3)$ \\
\hline$(11)$ & $\mathrm{SO}(18)$ & $\mathbf{2 5 6}$ & $\mathrm{SO}(10) \times \mathrm{U}(1)$ & $\mathbf{1 0}(0)$ & $\mathbf{1 6}(2), \mathbf{1 6}(-2), \overline{\mathbf{1 6}}(-2), \overline{\mathbf{1 6}}(2)$ \\
\hline$(11)$ & $\mathrm{SO}(19)$ & $\mathbf{5 1 2}$ & $\mathrm{SO}(10) \times \mathrm{U}(1)$ & $\mathbf{1 0}(0), \mathbf{1 0}(2), \mathbf{1 0}(-2)$ & $\mathbf{1 6}(1), \mathbf{1 6}(-1), \overline{\mathbf{1 6}}(1), \overline{\mathbf{1 6}}(-1)$ \\
\hline$(15)$ & $\mathrm{SO}(14)$ & $\mathbf{6 4}$ & $\mathrm{SO}(10) \times \mathrm{U}(1)$ & $(\mathrm{a}): \mathbf{1 0}(1), \mathbf{1 0}(-1), \mathbf{1}(2), \mathbf{1}(-2)$ & $(\mathrm{a}): \mathbf{1 6}(0), \mathbf{1 6}(1), \mathbf{1 6}(-1)$, \\
& & & & & $\overline{\mathbf{1 6}}(0), \overline{\mathbf{1 6}}(-1), \overline{\mathbf{1 6}}(1)$ \\
& & & & $(\mathrm{b}): \mathbf{1 0}(3), \mathbf{1 0}(-3)$ & $(\mathrm{b}): \mathbf{1 6}(0), \mathbf{1 6}(3), \mathbf{1 6}(-3)$, \\
& & & & & $\overline{\mathbf{1 6}}(0), \overline{\mathbf{1 6}}(-3), \overline{\mathbf{1 6}}(3)$ \\
\hline
\end{tabular}


Notice that there is another branch of the decomposition such as

$$
\begin{aligned}
\mathrm{SO}(19) & \supset \mathrm{SO}(18) \supset \mathrm{SO}(10) \times \mathrm{SO}(8) \\
& \supset \mathrm{SO}(10) \times \mathrm{SU}(2) \times \mathrm{SU}(2) \times \mathrm{SU}(2) \times \mathrm{SU}(2) \\
& \supset \mathrm{SO}(10) \times \mathrm{SU}(2) \times \mathrm{SU}(2) \times \mathrm{SU}(2) \times \mathrm{U}(1) .
\end{aligned}
$$

As mentioned at the beginning of this section, it gives different representations of the subgroup $\mathrm{SO}(10) \times \mathrm{SU}(2) \times \mathrm{SU}(2) \times \mathrm{SU}(2) \times \mathrm{U}(1)$ for a representation of $\mathrm{SO}(19)$. For example, the adjoint representation 171 of $\mathrm{SO}(19)$ is decomposed according to decomposition branch Eq. (14) and Eq. (15) as follows: $\left.{ }^{45)}, 46\right)$

$$
\begin{aligned}
\mathbf{1 7 1}= & (45, \mathbf{1}, \mathbf{1}, \mathbf{1})(0)+(\mathbf{1}, \mathbf{3}, \mathbf{1}, \mathbf{1})(0) \\
& +(\mathbf{1}, \mathbf{1}, \mathbf{3}, \mathbf{1})(0)+(\mathbf{1}, \mathbf{1}, \mathbf{1}, \mathbf{3})(0)+(\mathbf{1}, \mathbf{1}, \mathbf{1}, \mathbf{1})(0) \\
& +(\mathbf{1}, \mathbf{2}, \mathbf{2}, \mathbf{1})(2)+(\mathbf{1}, \mathbf{2}, \mathbf{2}, \mathbf{1})(-2)+(\mathbf{1}, \mathbf{2}, \mathbf{2}, \mathbf{3})(0) \\
& +(\mathbf{1}, \mathbf{1}, \mathbf{1}, \mathbf{3})(2)+(\mathbf{1}, \mathbf{1}, \mathbf{1}, \mathbf{3})(-2) \\
& +(\mathbf{1 0}, \mathbf{2}, \mathbf{2}, \mathbf{1})(0)+(\mathbf{1 0}, \mathbf{1}, \mathbf{1}, \mathbf{1})(2) \\
& +(\mathbf{1 0}, \mathbf{1}, \mathbf{1}, \mathbf{1})(-2)+(\mathbf{1 0}, \mathbf{1}, \mathbf{1}, \mathbf{3})(0), \\
\mathbf{1 7 1}= & (45, \mathbf{1}, \mathbf{1}, \mathbf{1})(0)+(\mathbf{1}, \mathbf{3}, \mathbf{1}, \mathbf{1})(0) \\
& +(\mathbf{1}, \mathbf{1}, \mathbf{3}, \mathbf{1})(0)+(\mathbf{1}, \mathbf{1}, \mathbf{1}, \mathbf{3})(0)+(\mathbf{1}, \mathbf{1}, \mathbf{1}, \mathbf{1})(0) \\
& +(\mathbf{1 0}, \mathbf{1}, \mathbf{1}, \mathbf{1})(0)+(\mathbf{1 0}, \mathbf{2}, \mathbf{2}, \mathbf{1})(0) \\
& +(\mathbf{1}, \mathbf{2}, \mathbf{2}, \mathbf{1})(0)+(\mathbf{1}, \mathbf{1}, \mathbf{1}, \mathbf{1})(2)+(\mathbf{1}, \mathbf{1}, \mathbf{1}, \mathbf{1})(-2) \\
& +(\mathbf{1}, \mathbf{2}, \mathbf{2}, \mathbf{2})(1)+(\mathbf{1}, \mathbf{2}, \mathbf{2}, \mathbf{2})(-1) \\
& +(\mathbf{1 0}, \mathbf{1}, \mathbf{1}, \mathbf{2})(1)+(\mathbf{1 0}, \mathbf{1}, \mathbf{1}, \mathbf{2})(-1) \\
& +(\mathbf{1}, \mathbf{1}, \mathbf{1}, \mathbf{2})(1)+(\mathbf{1}, \mathbf{1}, \mathbf{1}, \mathbf{2})(-1) .
\end{aligned}
$$

The singlets of $\mathrm{SU}(2) \times \mathrm{SU}(2) \times \mathrm{SU}(2) \times \mathrm{U}(1)$, which are $(\mathbf{4 5}, \mathbf{1}, \mathbf{1}, \mathbf{1})(0)$ and $(\mathbf{1 0}, \mathbf{1}, \mathbf{1}, \mathbf{1})(0)$, form an adjoint representation of $\mathrm{SO}(11)$ which is $(\mathbf{5 5}, \mathbf{1}, \mathbf{1}, \mathbf{1})(0)$. This indicates that the centralizer of $\mathrm{SU}(2) \times \mathrm{SU}(2) \times \mathrm{SU}(2) \times \mathrm{U}(1)$ is not $H=\mathrm{SO}(10) \times \mathrm{U}(1)$ but $\mathrm{SO}(11) \times \mathrm{U}(1)$, which is irrelevant to our purpose.

(b) $S / R(15 \mathrm{a})=\mathrm{G}_{2} / \mathrm{SU}(2) \times \mathrm{U}(1), G=\mathrm{SO}(14)$, and $F=\mathbf{6 4}$.

We embed $R$ in the subgroup $\mathrm{SU}(2) \times \mathrm{U}(1)$ of $G=\mathrm{SO}(14)$ according to the decomposition

$$
\begin{aligned}
\mathrm{SO}(14) & \supset \mathrm{SO}(10) \times \mathrm{SU}(2) \times \mathrm{SU}(2) \\
& \supset \mathrm{SO}(10) \times \mathrm{SU}(2) \times \mathrm{U}(1) .
\end{aligned}
$$


There are two branches of embedding which leads to the field contents of the SM in this case, owing to the freedom of the normalization of $\mathrm{U}(1)$ charges as mentioned in the beginning part of this section. For example, the adjoint representation of $\mathrm{SO}(14)$ can be decomposed according to Eq. (18) as ${ }^{45), 46)}$

$$
\begin{aligned}
\mathbf{9 1}= & (\mathbf{4 5}, \mathbf{1})(0)+(\mathbf{1}, \mathbf{3})(0)+(\mathbf{1}, \mathbf{1})(0) \\
& +(\mathbf{1}, \mathbf{1})(2 x)+(\mathbf{1}, \mathbf{1})(-2 x) \\
& +(\mathbf{1 0}, \mathbf{2})(x)+(\mathbf{1 0}, \mathbf{2})(-x),
\end{aligned}
$$

where $x$ is an arbitrary number reflecting the freedom of the normalization. The choice of $x=1$ and $x=3$ leads to the scalar contents (a) and (b) of Table $\mathrm{V}$ respectively, as can be seen by comparing the U(1) charges of Eq. (19) with those in the row (15a) of Table II.

(c) $S / R(1)=\mathrm{SO}(11) / \mathrm{SO}(10), G=\mathrm{SO}(20)$, and $F=\mathbf{5 1 2}$.

We embed $R$ in the subgroup $\mathrm{SO}(10)$ of $G=\mathrm{SO}(20)$ according to the decomposition

$$
\mathrm{SO}(20) \supset \mathrm{SO}(10) \times \mathrm{SO}(10)
$$

(d) $S / R(2)=\mathrm{SO}(7) \times \mathrm{Sp}(4) / \mathrm{SO}(6) \times[\mathrm{SU}(2) \times \mathrm{SU}(2)], G=\mathrm{SO}(20)$, and $F=\mathbf{5 1 2}$.

We embed $R$ in the subgroup $\mathrm{SU}(4) \times \mathrm{SU}(2) \times \mathrm{SU}(2)$ of $G=\mathrm{SO}(20)$ according to the decomposition

$$
\begin{aligned}
\mathrm{SO}(20) & \supset \mathrm{SO}(10) \times \mathrm{SO}(10) \\
& \supset \mathrm{SO}(10) \times \mathrm{SU}(4) \times \mathrm{SU}(2) \times \mathrm{SU}(2) .
\end{aligned}
$$

(e) $S / R(4)=\mathrm{SU}(6) / \mathrm{SU}(5) \times \mathrm{U}(1), G=\mathrm{SO}(20)$, and $F=\mathbf{5 1 2}$.

We embed $R$ in the subgroup $\mathrm{SU}(5) \times \mathrm{U}(1)$ of $G=\mathrm{SO}(20)$ according to the decomposition

$$
\begin{aligned}
\mathrm{SO}(20) & \supset \mathrm{SO}(10) \times \mathrm{SO}(10) \\
& \supset \mathrm{SO}(10) \times \mathrm{SU}(5) \times \mathrm{U}(1) .
\end{aligned}
$$

(f) $S / R(5)=\mathrm{SO}(9) \times \mathrm{SU}(2) / \mathrm{SO}(8) \times \mathrm{U}(1), G=\mathrm{SO}(20)$, and $F=\mathbf{5 1 2}$.

We embed $R$ in the subgroup $\mathrm{SO}(8) \times \mathrm{U}(1)$ of $G=\mathrm{SO}(20)$ according to the decomposition

$$
\begin{aligned}
\mathrm{SO}(20) & \supset \mathrm{SO}(10) \times \mathrm{SO}(10) \\
& \supset \mathrm{SO}(10) \times \mathrm{SO}(8) \times \mathrm{U}(1) .
\end{aligned}
$$

(g) $S / R(6)=\mathrm{SO}(7) \times \mathrm{SU}(3) / \mathrm{SO}(6) \times[\mathrm{SU}(2) \times \mathrm{U}(1)], G=\mathrm{SO}(20)$, and $F=\mathbf{5 1 2}$. 
We embed $R$ in the subgroup $\mathrm{SU}(4) \times \mathrm{SU}(2) \times \mathrm{U}(1)$ of $G=\mathrm{SO}(20)$ according to the decomposition

$$
\begin{aligned}
\mathrm{SO}(20) & \supset \mathrm{SO}(10) \times \mathrm{SO}(10) \\
& \supset \mathrm{SO}(10) \times \mathrm{SU}(4) \times \mathrm{SU}(2) \times \mathrm{SU}(2) \\
& \supset \mathrm{SO}(10) \times \mathrm{SU}(4) \times \mathrm{SU}(2) \times \mathrm{U}(1)
\end{aligned}
$$

(h) $S / R(8)=\{\mathrm{Sp}(4)\}^{2} \times \mathrm{SU}(2) /[\mathrm{SU}(2) \times \mathrm{SU}(2)]^{2} \times \mathrm{U}(1), G=\mathrm{SO}(20)$, and $F=\mathbf{5 1 2}$.

We embed $R$ in the subgroup $\mathrm{SU}(2) \times \mathrm{SU}(2) \times \mathrm{SU}(2) \times \mathrm{SU}(2) \times \mathrm{U}(1)$ of $G=\mathrm{SO}(20)$ according to the decomposition

$$
\begin{aligned}
\mathrm{SO}(20) & \supset \mathrm{SO}(10) \times \mathrm{SO}(10) \\
& \supset \mathrm{SO}(10) \times \mathrm{SU}(4) \times \mathrm{SU}(2) \times \mathrm{SU}(2) \\
& \supset \mathrm{SO}(10) \times \mathrm{SU}(2)^{\prime} \times \mathrm{SU}(2)^{\prime} \\
& \times \mathrm{SU}(2) \times \mathrm{SU}(2) \times \mathrm{U}(1)
\end{aligned}
$$

(i) $S / R(10)=\mathrm{Sp}(4) \times \mathrm{Sp}(4) /[\mathrm{SU}(2) \times \mathrm{U}(1)]_{\max } \times[\mathrm{SU}(2) \times \mathrm{SU}(2)], G=\mathrm{SO}(18)$, and $F=\mathbf{2 5 6}$.

We embed $R$ in the subgroup $\mathrm{SU}(2) \times \mathrm{SU}(2) \times \mathrm{SU}(2) \times \mathrm{U}(1)$ of $G=\mathrm{SO}(18)$ according to the decomposition

$$
\begin{aligned}
\mathrm{SO}(18) & \supset \mathrm{SO}(10) \times \mathrm{SO}(8) \\
& \supset \mathrm{SO}(10) \times \mathrm{SU}(2) \times \mathrm{SU}(2) \times \mathrm{SU}(2) \times \mathrm{SU}(2) \\
& \supset \mathrm{SO}(10) \times \mathrm{SU}(2) \times \mathrm{SU}(2) \times \mathrm{SU}(2) \times \mathrm{U}(1) .
\end{aligned}
$$

(j) $S / R(11)=\mathrm{Sp}(4) \times \mathrm{Sp}(4) /[\mathrm{SU}(2) \times \mathrm{U}(1)]_{\text {non-max }} \times[\mathrm{SU}(2) \times \mathrm{SU}(2)], G=\mathrm{SO}(18)$ and $F=256$.

We embed $R$ in the subgroup $\mathrm{SU}(2) \times \mathrm{SU}(2) \times \mathrm{SU}(2) \times \mathrm{U}(1)$ of $G=\mathrm{SO}(18)$ according to the decomposition

$$
\begin{aligned}
\mathrm{SO}(18) & \supset \mathrm{SO}(10) \times \mathrm{SO}(8) \\
& \supset \mathrm{SO}(10) \times \mathrm{SU}(2) \times \mathrm{SU}(2) \times \mathrm{SU}(2) \times \mathrm{SU}(2) \\
& \supset \mathrm{SO}(10) \times \mathrm{SU}(2) \times \mathrm{SU}(2) \times \mathrm{SU}(2) \times \mathrm{U}(1) .
\end{aligned}
$$

We find ten candidates of $(S / R, G, F)$ which give at least one fermion with representation 16 and scalar with 10 in four dimensions. Other combinations of $(S / R, G, F)$ are excluded since they do not provide both a representation $\mathbf{1 6}$ for fermions and a representation 10 for scalars. 
In many cases we obtain several 16s for fermions. Particularly interesting candidates among them are $(G=\mathrm{SO}(20), S / R(4), F=\mathbf{5 1 2})$ and $(G=\mathrm{SO}(20), S / R(6), F=\mathbf{5 1 2})$. They give three 16s corresponding to three generations of fermions. In such cases the extra $\mathrm{U}(1)$ symmetry can be interpreted as a family symmetry.

We obtain the scalar field in the $\mathbf{1 0}$ representation of $\mathrm{SO}(10)$ in all cases. This scalar field contains the SM Higgs. Notice, however, that no scalar content belongs to $\mathbf{1 6}, \mathbf{4 5}, \mathbf{1 2 6}, \cdots$, which are necessary to break $\mathrm{SO}(10)$ to the $\mathrm{SM}$ gauge group. This is inevitable for $H=$ $\mathrm{SO}(10)(\times \mathrm{U}(1))$. The gauge group $G$ for $H=\mathrm{SO}(10)(\times \mathrm{U}(1))$ is $\mathrm{SO}(N)$, and $\mathrm{SO}(10)$ appears in the decomposition

$$
\mathrm{SO}(N) \supset \mathrm{SO}(10) \times \mathrm{SO}(N-10) \supset \cdots .
$$

Only 1 or 10 representations of $\mathrm{SO}(10)$ are obtained from the adjoint representation of $\mathrm{SO}(N)$ under the above decomposition. Thus no scalar can break $\mathrm{SO}(10)$ to the SM gauge group. Fortunately, we can construct a phenomenologically acceptable model without these scalar contents by employing the topological symmetry breaking mechanism, known as Hosotani mechanism or Wilson flux breaking mechanism. ${ }^{29), 30), 47)-53)}$ This mechanism requires extra-dimensional spaces to be non-simply connected. Hence we have to consider the non-simply connected coset spaces such as $(S / R) / T$ instead of the simply connected ones, where $T$ is a suitable discrete symmetry group.

\section{2. $H=\mathrm{SU}(5) \times \mathrm{U}(1)$}

Secondly, we search for viable $\mathrm{SU}(5) \times \mathrm{U}(1)$ models in four dimensions. We list below the combinations of $S / R, G$ and $F$ which provides $H=\mathrm{SU}(5) \times \mathrm{U}(1)$ and representations which contain field contents of the SM for the scalars and the fermions. The embedding of $R$ into $G$ is shown for each candidates since this embedding uniquely determines all the representations of the scalars and fermions in the four-dimensional theory. In Table VI, we show all the field contents in four dimensions for each combination of $(S / R, G, F)$.

(a) $S / R(15)=\mathrm{G}_{2} / \mathrm{SU}(2) \times \mathrm{U}(1), G=\mathrm{Sp}(12)$ and $F=\mathbf{2 0 8}$.

We embed $R$ in the subgroup $\mathrm{SU}(2) \times \mathrm{U}(1)$ of $G=\mathrm{Sp}(12)$ according to the decomposition

$$
\begin{aligned}
\mathrm{Sp}(12) & \supset \mathrm{Sp}(10) \times \mathrm{Sp}(2) \\
& \supset \mathrm{SU}(5) \times \mathrm{SU}(2) \times \mathrm{U}(1) .
\end{aligned}
$$

(b) $S / R(14)=\operatorname{Sp}(6) / \mathrm{Sp}(4) \times \mathrm{U}(1), G=\mathrm{Sp}(14)$, and $F=\mathbf{3 5 0}$.

We embed $R$ in the subgroup $\operatorname{Sp}(4) \times \mathrm{U}(1)$ of $G=\operatorname{Sp}(14)$ according to the decomposition

$$
\begin{aligned}
\mathrm{Sp}(14) & \supset \mathrm{Sp}(10) \times \mathrm{Sp}(4) \\
& \supset \mathrm{SU}(5) \times \mathrm{Sp}(4) \times \mathrm{U}(1)
\end{aligned}
$$


Table VI. The field contents in four dimensions with $H=\mathrm{SU}(5) \times \mathrm{U}(1)$ for each combination of $(S / R, G, F)$. Coset spaces are indicated by the number assigned in Table 【.

\begin{tabular}{l|l|l||l|l}
\hline \multicolumn{2}{l||}{$14 \mathrm{D}$ model } & 4D model \\
\hline$S / R$ & $G$ & $F$ & Scalars & Fermions \\
\hline$(11)$ & $\operatorname{Sp}(16)$ & $\mathbf{5 4 4}$ & $\begin{array}{l}\mathbf{1 5}(2), \overline{\mathbf{1 5}}(-2), \mathbf{5}(1), \overline{\mathbf{5}}(-1), \\
\mathbf{1}(0)\end{array}$ & $\begin{array}{l}\{\mathbf{2 4}(0)\}^{2}, \mathbf{1 0}(2), \overline{\mathbf{1 0}}(-2), \mathbf{5}(1), \\
\overline{\mathbf{5}}(-1),\{\mathbf{1}(0)\}^{4}\end{array}$ \\
\hline$(14)$ & $\mathrm{Sp}(14)$ & $\mathbf{3 5 0}$ & $\mathbf{1 5}(-2), \overline{\mathbf{1 5}}(2), \mathbf{5}(-1), \overline{\mathbf{5}}(1)$ & $\begin{array}{l}\mathbf{4 5}(1), \overline{\mathbf{4 5}}(-1), \mathbf{2 4}(0), \mathbf{1 0}(3), \\
\mathbf{1 0}(-2), \overline{\mathbf{5}}(1), \mathbf{5}(1), \overline{\mathbf{5}}(-1)\end{array}$ \\
\hline$(15)$ & $\mathrm{Sp}(12)$ & $\mathbf{2 0 8}$ & $\mathbf{1 5}(2), \overline{\mathbf{1 5}}(-2), \mathbf{5}(1), \overline{\mathbf{5}}(-1)$ & $\begin{array}{l}\mathbf{4 5}(1), \overline{\mathbf{4 5}}(-1), \mathbf{2 4}(0), \mathbf{1 0}(-3), \\
\overline{\mathbf{1 0}}(3), \mathbf{1 0}(2), \overline{\mathbf{1 0}}(-2), \mathbf{5}(1), \overline{\mathbf{5}}(-1)\end{array}$ \\
\hline
\end{tabular}

(c) $S / R(11)=\mathrm{Sp}(4) \times \mathrm{Sp}(4) /[\mathrm{SU}(2) \times \mathrm{U}(1)]_{\text {non-max }} \times[\mathrm{SU}(2) \times \mathrm{SU}(2)], G=\mathrm{Sp}(16)$, and $F=544$.

We embed $R$ in the subgroup $\mathrm{SU}(2)^{\prime} \times \mathrm{SU}(2)^{\prime} \times \mathrm{SU}(2) \times \mathrm{U}(1)$ of $G=\mathrm{Sp}(16)$ according to the decomposition

$$
\begin{aligned}
\mathrm{Sp}(16) & \supset \mathrm{Sp}(10) \times \mathrm{Sp}(6) \\
& \supset \mathrm{Sp}(10) \times \mathrm{Sp}(4) \times \mathrm{SU}(2) \\
& \supset \mathrm{Sp}(10) \times \mathrm{SU}(2)^{\prime} \times \mathrm{SU}(2)^{\prime} \times \mathrm{SU}(2) \\
& \supset \mathrm{SU}(5) \times \mathrm{SU}(2)^{\prime} \times \mathrm{SU}(2)^{\prime} \times \mathrm{SU}(2) \times \mathrm{U}(1)
\end{aligned}
$$

We find three candidates of $(S / R, G, F)$ that give at least one pair of fermions with representation 10 and $\overline{\mathbf{5}}$, and a scalar with $\mathbf{5}$ representation in four dimensions. Other combinations of $(S / R, G, F)$ are excluded since they do not provide these representations for fermions and scalars.

We obtain the scalar field in 5 representation of $\mathrm{SU}(5)$ for all cases. This scalar field contains the SM Higgs. Notice, however, that no scalar contents belongs to $\mathbf{2 4}, \cdots$, which are necessary to break $\mathrm{SU}(5)$ to the SM gauge group. The lack of such scalars is a general feature for $H=\mathrm{SU}(5) \times \mathrm{U}(1)$. The gauge groups $G$ for $H=\mathrm{SU}(5) \times \mathrm{U}(1)$ are $\mathrm{SU}(N)$, $\mathrm{SO}(N)$, and $\mathrm{Sp}(N)$. These groups are decomposed into subgroups including $\mathrm{SU}(5) \times \mathrm{U}(1)$, and their adjoint representations are decomposed accordingly as well:

$$
\begin{aligned}
& \mathrm{SU}(N) \supset \mathrm{SU}(5) \times \mathrm{SU}(N-5) \times \mathrm{U}(1) \supset \cdots \\
& \operatorname{adj} \mathrm{SU}(5)=(\mathbf{2 4}, \mathbf{1})(0)+(\mathbf{1}, \operatorname{adj} \mathrm{SU}(N-1))(0)+(1,1)(0) \\
&+(\mathbf{5}, \overline{N-5})(a)+(\overline{\mathbf{5}}, N-5)(-a) \\
&= \cdots
\end{aligned}
$$




$$
\begin{aligned}
& \mathrm{SO}(N) \supset \mathrm{SO}(10) \times \mathrm{SO}(N-10) \\
& \supset \mathrm{SU}(5) \times \mathrm{SO}(N-10) \times \mathrm{U}(1) \supset \cdots \\
& \operatorname{adj} \mathrm{SO}(N)=(\mathbf{4 5}, \mathbf{1})+(\mathbf{1}, \operatorname{adj} \mathrm{SO}(N-10)) \\
&+(\mathbf{1 0}, \mathbf{1})+(\mathbf{1}, N-10) \\
&=(\mathbf{2 4}, \mathbf{1})(0)+(\mathbf{1}, \operatorname{adj} \mathrm{SO}(N-10))(0)+(\mathbf{1}, \mathbf{1})(0) \\
&+(\mathbf{1 0}, \mathbf{1})(4)+(\overline{\mathbf{1 0}}, \mathbf{1})(-4)+(\mathbf{5}, \mathbf{1})(2)+(\overline{\mathbf{5}}, \mathbf{1})(-2)+(\mathbf{1}, N-10)(0) \\
&= \cdots \\
& \mathrm{Sp}(2 N) \supset \mathrm{Sp}(10) \times \mathrm{Sp}(2 N-10) \\
& \supset \mathrm{SU}(\mathbf{5}) \times \mathrm{Sp}(2 N-1) \times \mathrm{U}(1) \\
& \supset \cdots \\
& \operatorname{adj} \operatorname{Sp}(2 N)=(\mathbf{5 5}, \mathbf{1})+(\mathbf{1}, \operatorname{adj} \operatorname{Sp}(2 N-10)) \\
&+(\mathbf{1 0}, \mathbf{1})+(\mathbf{1}, 2 N-10) \\
&=(\mathbf{2 4}, \mathbf{1})(0)+(\mathbf{1}, \operatorname{adj} \operatorname{Sp}(2 N-10))(0)+(\mathbf{1}, \mathbf{1})(0) \\
&+(\mathbf{1 5}, \mathbf{1})(2)+(\overline{\mathbf{1 5}}, \mathbf{1})(-2)+(\mathbf{5}, \mathbf{1})(1)+(\overline{\mathbf{5}}, \mathbf{1})(-1)+(\mathbf{1}, N)(0) \\
&=\cdots .
\end{aligned}
$$

Only 1, 5, 10, or $\mathbf{1 5}$ representation of $\mathrm{SU}(5)$ is obtained from the adjoint representations of $\mathrm{SU}(N), \mathrm{SO}(N)$, and $\mathrm{Sp}(N)$ under the above decompositions. Then, no scalar can break $\mathrm{SU}(5)$ to the SM gauge group. Therefore we should employ the flux breaking mechanism to break $\mathrm{SU}(5)$ to the SM gauge group.

\section{3. $H=\mathrm{SU}(3) \times \mathrm{SU}(2) \times \mathrm{U}(1)$}

We find no viable candidate for $H=\mathrm{SU}(3) \times \mathrm{SU}(2) \times \mathrm{U}(1)$. We exclude the coset spaces (16) - (35) in Table I. They have two or more factors of U(1) in $R$, and these U(1)'s become the part of $H=C_{G}(R)=\mathrm{SU}(3) \times \mathrm{SU}(2) \times \mathrm{U}(1)$, which has only one $\mathrm{U}(1)$. The single $\mathrm{U}(1)$ factor in $R$ becomes $\mathrm{U}(1)_{Y}$ of the SM gauge group, hence the decomposition of the spinor representation 16 of $\mathrm{SO}(10)$ to $R$ need to have $\mathrm{U}(1)$ charges whose ratio is $1: 2:(-3):(-4): 6$. Referring to Table III, we find that the coset spaces $(4)-(15)$ do not have such $\mathrm{U}(1)$ charge and thus are excluded. The explicit analysis of the remaining coset spaces (1), (2) and (3) shows that they do not induce the SM either.

3.4. $H=\mathrm{SU}(3) \times \mathrm{SU}(2) \times \mathrm{U}(1) \times \mathrm{U}(1)$

Finally, we search for viable $\mathrm{SU}(3) \times \mathrm{SU}(2) \times \mathrm{U}(1) \times \mathrm{U}(1)$ models in four dimensions. We list below the combinations of $S / R, G$, and $F$ which provide $H=\mathrm{SU}(3) \times \mathrm{SU}(2) \times$ $\mathrm{U}(1) \times \mathrm{U}(1)$ and representations of the SM scalars and fermions. Embedding of $R$ in $G$ 
is also shown for each candidates. Note that we can take a linear combination of the two $\mathrm{U}(1)$ 's. The U(1) charges in the decomposition are first chosen to facilitate the decomposition of the group $G$, then combined to embed $R$ into $G$, and subsequently organized again to reproduce the hypercharge of the SM. We explicitly show these linear recombinations of U(1) for each candidates. In Table VIII, we show all the field contents in four dimensions for each combination of $(S / R, G, F)$.

Table VII. The field contents in four dimensions with $H=\mathrm{SU}(3) \times \mathrm{SU}(2) \times \mathrm{U}(1)_{R} \times \mathrm{U}(1)_{A}$. Coset spaces are indicated by the number assigned in Table \. Numbers in a superscript of the representations denote its multiplicity.

\begin{tabular}{|c|c|c|c|c|}
\hline \multicolumn{3}{|c|}{ 14D model } & \multicolumn{2}{|l|}{ 4D model } \\
\hline$S / R$ & $G$ & $F$ & Scalars & Fermions \\
\hline$(15 a)$ & $\mathrm{Sp}(12)$ & 364 & $\begin{array}{l}(\mathbf{1}, \mathbf{2})(-2,3),(\mathbf{1}, \mathbf{2})(2,-3), \\
(\mathbf{3}, \mathbf{1})(-1,-4),(\overline{\mathbf{3}}, \mathbf{1})(1,4) \\
(\mathbf{6}, \mathbf{1})(-2,-8),(\overline{\mathbf{6}}, \mathbf{1})(2,8)\end{array}$ & $\begin{array}{l}(\mathbf{1 5}, \mathbf{1})(-1,4),(\overline{\mathbf{1 5}}, \mathbf{1})(1,-4),(\mathbf{1 0}, \mathbf{1})(-3,-12), \\
(\overline{\mathbf{1 0}}, \mathbf{1})(3,12),(\mathbf{3}, \mathbf{1})(-1,-4),\{(\overline{\mathbf{3}}, \mathbf{1})(1,4)\}^{3}, \\
(\mathbf{1}, \mathbf{3})(0,0),(\mathbf{1}, \mathbf{1})(-4,6),\{(\mathbf{1}, \mathbf{1})(0,0)\}^{2}, \\
(\mathbf{1}, \mathbf{2})(-2,3),(\mathbf{1}, \mathbf{2})(2,-3),(\mathbf{3}, \mathbf{3})(-1,-4), \\
(\overline{\mathbf{3}}, \mathbf{3})(1,4),(\overline{\mathbf{3}}, \mathbf{1})(5,-2),(\mathbf{3}, \mathbf{1})(-1,-4), \\
(\mathbf{3}, \mathbf{1})(3,-10),(\overline{\mathbf{3}}, \mathbf{1})(-3,10),(\mathbf{3}, \mathbf{2})(-3,-1), \\
(\overline{\mathbf{3}}, \mathbf{2})(3,1),(\mathbf{3}, \mathbf{2})(1,-7),(\overline{\mathbf{3}}, \mathbf{2})(-1,7), \\
(\mathbf{8}, \mathbf{1})(0,0),(\mathbf{6}, \mathbf{1})(2,-8),(\overline{\mathbf{6}}, \mathbf{1})(-2,8)\end{array}$ \\
\hline (9) & $\mathrm{Sp}(16)$ & 544 & $(\mathbf{1}, \mathbf{2})(1,0),(\mathbf{1}, \mathbf{2})(-1,0)$ & $\begin{array}{l}(\mathbf{1}, \mathbf{1})(-2,0),(\mathbf{1}, \mathbf{2})(1,0),\{(\mathbf{1}, \mathbf{1})(0,0)\}^{2} \\
(\overline{\mathbf{3}}, \mathbf{1})(2,-1),(\mathbf{3}, \mathbf{1})(2,1),(\overline{\mathbf{3}}, \mathbf{2})(-1,-1) \\
(\mathbf{3}, \mathbf{2})(-1,1),\{(\mathbf{3}, \mathbf{1})(0,1)\}^{3},\{(\overline{\mathbf{3}}, \mathbf{1})(0,-1)\}^{3} \\
(\mathbf{8}, \mathbf{1})(0,0),(\mathbf{6}, \mathbf{1})(0,-1),(\overline{\mathbf{6}}, \mathbf{1})(0,1)\end{array}$ \\
\hline$(15 \mathrm{a})$ & $\mathrm{SO}(13)$ & 768 & $\begin{array}{l}(\mathbf{1}, \mathbf{2})(3,3),(\mathbf{1}, \mathbf{2})(-3,-3) \\
(\mathbf{3}, \mathbf{1})(-2,-6),(\overline{\mathbf{3}}, \mathbf{1})(2,6)\end{array}$ & $\begin{array}{l}(\mathbf{3}, \mathbf{3})(-2,-4),(\overline{\mathbf{3}}, \mathbf{3})(2,4),(\mathbf{1}, \mathbf{3})(0,-6),(\mathbf{1}, \mathbf{3})(0,6), \\
(\mathbf{3}, \mathbf{2})(1,3),(\overline{\mathbf{3}}, \mathbf{1})(-4,-6),(\mathbf{3}, \mathbf{1})(-2,0),(\overline{\mathbf{3}}, \mathbf{1})(2,0), \\
(\mathbf{3}, \mathbf{2})(1,3),(\overline{\mathbf{3}}, \mathbf{2})(-1,-3),(\overline{\mathbf{3}}, \mathbf{2})(5,3),(\mathbf{1}, \mathbf{1})(0,-6), \\
(\mathbf{1}, \mathbf{1})(0,6),(\mathbf{1}, \mathbf{2})(3,-3),(\mathbf{1}, \mathbf{2})(-3,3), \\
(\mathbf{1}, \mathbf{2})(-3,-9),(\mathbf{1}, \mathbf{2})(3,9),(\mathbf{3}, \mathbf{2})(1,3), \\
(\overline{\mathbf{3}}, \mathbf{2})(-1,-3),(\mathbf{3}, \mathbf{1})(-2,0),(\overline{\mathbf{3}}, \mathbf{1})(2,0), \\
(\mathbf{1}, \mathbf{1})(0,6),(\mathbf{1}, \mathbf{1})(0,-6),(\mathbf{1}, \mathbf{2})(3,-3),(\mathbf{1}, \mathbf{2})(-3,3), \\
(\mathbf{3}, \mathbf{1})(-2,0),(\overline{\mathbf{3}}, \mathbf{1})(2,0),(\mathbf{3}, \mathbf{2})(1,3),(\overline{\mathbf{3}}, \mathbf{2})(-1,-3), \\
(\overline{\mathbf{3}}, \mathbf{1})(-4,6),(\mathbf{3}, \mathbf{2})(1,-9),(\overline{\mathbf{3}}, \mathbf{2})(-1,9),(\mathbf{6}, \mathbf{1})(2,0), \\
(\overline{\mathbf{6}}, 1)(-2,0),(\mathbf{6}, \mathbf{2})(-1,-3),(\overline{6}, 2)(1,3),(\mathbf{8}, \mathbf{1})(2,0), \\
(\mathbf{8}, \mathbf{1})(-2,0),(\mathbf{8}, \mathbf{2})(-1,-3),(\mathbf{8}, \mathbf{2})(1,3), \\
(\mathbf{3}, \mathbf{1})(-2,0),(\overline{\mathbf{3}}, \mathbf{1})(2,0),(\mathbf{3}, \mathbf{2})(1,3),(\overline{\mathbf{3}}, \mathbf{2})(-1,-3), \\
(\mathbf{1}, \mathbf{1})(0,-6),(\mathbf{1}, \mathbf{1})(0,6),(\mathbf{1}, \mathbf{2})(3,-3),(\mathbf{1}, \mathbf{2})(-3,3)\end{array}$ \\
\hline$(14)$ & $\mathrm{Sp}(14)$ & 350 & $\begin{array}{l}(\mathbf{1}, \mathbf{2})(-1,-9 / 2) \\
(\mathbf{1}, \mathbf{2})(1,9 / 2) \\
(\mathbf{3}, \mathbf{2})(-2,11 / 2) \\
(\overline{\mathbf{3}}, \mathbf{2})(2,-11 / 2) \\
(\mathbf{1}, \mathbf{3})(-2,-9),(\mathbf{1}, \mathbf{3})(2,9) \\
\end{array}$ & $\begin{array}{l}(\mathbf{6}, \mathbf{1})(3,-1),(\mathbf{8}, \mathbf{1})(0,0),(\mathbf{1}, \mathbf{1})(-2,-9), \\
\{(\mathbf{1}, \mathbf{1})(0,0)\}^{2},(\mathbf{3}, \mathbf{1})(-1,10),(\overline{\mathbf{3}}, \mathbf{1})(1,-10), \\
\{(\overline{\mathbf{3}}, \mathbf{1})(3,-1)\}^{2},\{(\mathbf{1}, \mathbf{2})(-1,-9 / 2)\}^{2}, \\
\{(\mathbf{1}, \mathbf{2})(1,9 / 2)\}^{3},(\mathbf{3}, \mathbf{2})(-2,11 / 2),(\mathbf{1}, \mathbf{3})(0,0), \\
(\overline{\mathbf{3}}, \mathbf{3})(3,-1)\end{array}$ \\
\hline
\end{tabular}

(a) $S / R(15 \mathrm{a})=\mathrm{G}_{2} / \mathrm{SU}(2) \times \mathrm{U}(1), G=\mathrm{Sp}(12)$, and $F=\mathbf{3 6 4}$.

We decompose $\operatorname{Sp}(12)$ as

$$
\begin{aligned}
\mathrm{Sp}(12) & \supset \mathrm{Sp}(6) \times \mathrm{Sp}(6) \\
& \supset \mathrm{Sp}(6) \times \mathrm{Sp}(4) \times \mathrm{SU}(2)^{\prime}
\end{aligned}
$$


Table VIII. The field contents in four dimensions with $H=\mathrm{SU}(3) \times \mathrm{SU}(2) \times \mathrm{U}(1)_{Y} \times \mathrm{U}(1)_{\alpha}$. Coset spaces are indicated by the number assigned in Table I. Numbers in superscript of the representations denote its multiplicity. The U(1) charges are rearranged from those of TableVII so that the charge of $\mathrm{U}(1)_{Y}$ is proportional to the hypercharge of the Standard Model.

\begin{tabular}{|c|c|c|c|c|c|c|}
\hline \multicolumn{3}{|c|}{$14 \mathrm{D}$ model } & \multicolumn{4}{|l|}{ 4D model } \\
\hline \multirow[b]{2}{*}{$S / R$} & \multirow[b]{2}{*}{$G$} & \multirow[b]{2}{*}{$F$} & \multicolumn{2}{|l|}{ Scalars } & \multicolumn{2}{|l|}{ Fermions } \\
\hline & & & SM fields & Extra fields & SM fields & Extra fields \\
\hline$(15 \mathrm{a})$ & $\mathrm{Sp}(12)$ & 364 & $\begin{array}{l}(\mathbf{1}, \mathbf{2})(3,-32) \\
(\mathbf{1}, \mathbf{2})(-3,32)\end{array}$ & $\begin{array}{l}(\mathbf{3}, \mathbf{1})(-2,-27) \\
(\overline{\mathbf{3}}, \mathbf{1})(2,27), \\
(\mathbf{6}, \mathbf{1})(-4,-54), \\
(\overline{\mathbf{6}}, \mathbf{1})(4,54)\end{array}$ & $\begin{array}{l}(\mathbf{3}, \mathbf{2})(1,-59) \\
(\overline{\mathbf{3}}, \mathbf{1})(2,27) \\
(\overline{\mathbf{3}}, \mathbf{1})(-4,91) \\
(\mathbf{1}, \mathbf{2})(-3,32) \\
(\mathbf{1}, \mathbf{1})(6,-64)\end{array}$ & $\begin{array}{l}(\mathbf{1 5}, 1)(34 / 11,-11), \\
(\overline{\mathbf{1 5}}, \mathbf{1})(-34 / 11,11), \\
(\mathbf{1 0}, \mathbf{1})(-6,-81),(\overline{\mathbf{1 0}}, \mathbf{1})(6,81), \\
\{(\mathbf{3}, \mathbf{1})(-2,-27)\}^{2},(\mathbf{1}, \mathbf{3})(0,0), \\
\{(\mathbf{1}, \mathbf{1})(0,0)\}^{2},(\mathbf{1}, \mathbf{2})(3,-32), \\
(\mathbf{3}, \mathbf{3})(-2,-27),(\overline{\mathbf{3}}, \mathbf{3})(2,27), \\
(\mathbf{3}, \mathbf{1})(-8,37),(\overline{\mathbf{3}}, \mathbf{1})(8,-37), \\
(\overline{\mathbf{3}}, \mathbf{2})(-1,59),(\mathbf{3}, \mathbf{2})(-5,5), \\
(\overline{\mathbf{3}}, \mathbf{2})(5,-5),(\mathbf{8}, \mathbf{1})(0,0), \\
\{(\overline{\mathbf{3}}, \mathbf{1})(2,27)\}^{2}, \\
(\mathbf{6}, \mathbf{1})(-68 / 11,22), \\
(\overline{\mathbf{6}}, \mathbf{1})(68 / 11,-22)\end{array}$ \\
\hline (9) & $\mathrm{Sp}(16)$ & 544 & $\begin{array}{l}(\mathbf{1}, \mathbf{2})(3,-2), \\
(\mathbf{1}, \mathbf{2})(-3,2)\end{array}$ & & $\begin{array}{l}(\mathbf{1}, \mathbf{1})(6,-4) \\
(\mathbf{1}, \mathbf{2})(-3,2) \\
(\overline{\mathbf{3}}, \mathbf{1})(-4,7) \\
(\overline{\mathbf{3}}, \mathbf{1})(2,3) \\
(\mathbf{3}, \mathbf{2})(1,-5)\end{array}$ & $\begin{array}{l}\{(\mathbf{1}, \mathbf{1})(0,0)\}^{2},(\mathbf{3}, \mathbf{1})(-8,1), \\
\{(\mathbf{3}, \mathbf{1})(-2,-3)\}^{3},\{(\overline{\mathbf{3}}, \mathbf{1})(2,3)\}^{2}, \\
(\overline{\mathbf{3}}, \mathbf{2})(5,1),(\mathbf{8}, \mathbf{1})(0,0), \\
(\mathbf{6}, \mathbf{1})(2,3),(\overline{\mathbf{6}}, \mathbf{1})(-2,-3)\end{array}$ \\
\hline$(15 \mathrm{a})$ & $\mathrm{SO}(13)$ & 768 & $\begin{array}{l}(\mathbf{1}, \mathbf{2})(-3,66), \\
(\mathbf{1}, \mathbf{2})(3,-66)\end{array}$ & $\begin{array}{l}(\mathbf{3}, \mathbf{2})(1,34) \\
(\overline{\mathbf{3}}, \mathbf{1})(2,100) \\
(\overline{\mathbf{3}}, \mathbf{1})(-4,32) \\
(\mathbf{1}, \mathbf{2})(-3,-102), \\
(\mathbf{1}, \mathbf{1})(6,36) \\
(\mathbf{3}, \mathbf{3})(0,8) \\
(\overline{\mathbf{3}}, \mathbf{3})(0,-8) \\
(\mathbf{1}, \mathbf{3})(-6,-36) \\
(\mathbf{1}, \mathbf{3})(6,36) \\
(\mathbf{3}, \mathbf{1})(4,-32) \\
(\overline{\mathbf{3}}, \mathbf{2})(-1,-34) \\
(\overline{\mathbf{3}}, \mathbf{2})(-7,98)\end{array}$ & $\begin{array}{l}(\mathbf{1}, \mathbf{1})(-6,-36), \\
(\mathbf{1}, \mathbf{2})(-9,30), \\
(\mathbf{1}, \mathbf{2})(9,-30), \\
(\mathbf{1}, \mathbf{2})(3,102), \\
(\overline{\mathbf{3}}, \mathbf{2})(-1,-34), \\
(\mathbf{3}, \mathbf{1})(4,-32), \\
(\mathbf{1}, \mathbf{1})(-6,-36)\end{array}$ & $\begin{array}{l}(\mathbf{1}, \mathbf{2})(-9,30),(\mathbf{1}, \mathbf{2})(9,-30), \\
(\mathbf{3}, \mathbf{1})(4,-32),(\overline{\mathbf{3}}, \mathbf{2})(-1,-34), \\
(\mathbf{3}, \mathbf{2})(-11,38),(\overline{\mathbf{3}}, \mathbf{2})(11,-38), \\
(\mathbf{6}, \mathbf{1})(-4,32),(\overline{\mathbf{6}}, \mathbf{1})(4,-32), \\
(\mathbf{6}, \mathbf{2})(-1,-34),(\overline{\mathbf{6}}, \mathbf{2})(1,34), \\
(\mathbf{8}, \mathbf{1})(-4,32),(\mathbf{8}, \mathbf{1})(4,-32), \\
(\mathbf{8}, \mathbf{2})(-1,-34),(\mathbf{8}, \mathbf{2})(1,34), \\
(\mathbf{3}, \mathbf{1})(4,-32),(\overline{\mathbf{3}}, \mathbf{2})(-1,-34), \\
(\mathbf{1}, \mathbf{1})(-6,-36),(\mathbf{1}, \mathbf{2})(-9,30), \\
(\mathbf{1}, \mathbf{2})(9,-30),(\overline{\mathbf{3}}, \mathbf{1})(2,100), \\
\{(\mathbf{3}, \mathbf{2})(1,34)\}^{5}, \\
\{(\overline{\mathbf{3}}, \mathbf{1})(-4,32)\}^{2},\{(\mathbf{1}, \mathbf{1})(6,36)\}^{2}\end{array}$ \\
\hline$(14)$ & $\mathrm{Sp}(14)$ & 350 & $\begin{array}{l}(\mathbf{1}, \mathbf{2})(3,-2), \\
(\mathbf{1}, \mathbf{2})(-3,2)\end{array}$ & & $\begin{array}{l}(\mathbf{1}, \mathbf{1})(6,-4), \\
(\mathbf{1}, \mathbf{2})(-3,2), \\
(\overline{\mathbf{3}}, \mathbf{1})(-4,7) \\
(\overline{\mathbf{3}}, \mathbf{1})(2,3) \\
(\mathbf{3}, \mathbf{2})(1,-5)\end{array}$ & $\begin{array}{l}\{(\mathbf{1}, \mathbf{1})(0,0)\}^{2},(\mathbf{3}, \mathbf{1})(-8,1), \\
\{(\mathbf{3}, \mathbf{1})(-2,-3)\}^{3},\{(\overline{\mathbf{3}}, \mathbf{1})(2,3)\}^{2}, \\
(\overline{\mathbf{3}}, \mathbf{2})(5,1),(\mathbf{8}, \mathbf{1})(0,0), \\
(\mathbf{6}, \mathbf{1})(2,3),(\overline{\mathbf{6}}, \mathbf{1})(-2,-3)\end{array}$ \\
\hline
\end{tabular}

$$
\begin{aligned}
& \supset \mathrm{SU}(3) \times \mathrm{Sp}(4) \times \mathrm{SU}(2)^{\prime} \times \mathrm{U}(1)_{\mathrm{a}} \\
& \supset \mathrm{SU}(3) \times \mathrm{SU}(2) \times \mathrm{SU}(2) \times \mathrm{SU}(2)^{\prime} \times \mathrm{U}(1)_{\mathrm{a}} \\
& \supset \mathrm{SU}(3) \times \mathrm{SU}(2) \times \mathrm{SU}(2)^{\prime} \times \mathrm{U}(1)_{\mathrm{a}} \times \mathrm{U}(1)_{\mathrm{b}} .
\end{aligned}
$$

Accordingly the adjoint representation of $\operatorname{Sp}(12)$ is decomposed as ${ }^{45), 46)}$

$$
\begin{aligned}
\mathbf{7 8}= & (\mathbf{8}, \mathbf{1}, \mathbf{1})(0,0)+(\mathbf{1}, \mathbf{3}, \mathbf{1})(0,0)+(\mathbf{1}, \mathbf{1}, \mathbf{3})(0,0)+(\mathbf{1}, \mathbf{1}, \mathbf{1})(0,0) \\
& +(\mathbf{1}, \mathbf{1}, \mathbf{1})(0,0)+(\mathbf{6}, \mathbf{1}, \mathbf{1})(2,0)+(\overline{\mathbf{6}}, \mathbf{1}, \mathbf{1})(-2,0)+(\mathbf{3}, \mathbf{1}, \mathbf{2})(1,0)
\end{aligned}
$$




$$
\begin{aligned}
& +(\overline{\mathbf{3}}, \mathbf{1}, \mathbf{2})(-1,0)+(\mathbf{3}, \mathbf{2}, \mathbf{1})(1,0)+(\overline{\mathbf{3}}, \mathbf{2}, 1)(-1,0)+(\mathbf{3}, \mathbf{1}, \mathbf{1})(1,1) \\
& +(\overline{\mathbf{3}}, \mathbf{1}, \mathbf{1})(-1,-1)+(\mathbf{3}, \mathbf{1}, \mathbf{1})(1,-1)+(\overline{\mathbf{3}}, \mathbf{1}, \mathbf{1})(-1,1)+(\mathbf{1}, \mathbf{2}, \mathbf{1})(0,1) \\
& +(\mathbf{1}, \mathbf{2}, \mathbf{1})(0,-1)+(\mathbf{1}, \mathbf{1}, \mathbf{2})(0,1)+(\mathbf{1}, \mathbf{1}, \mathbf{2})(0,-1)+(\mathbf{1}, \mathbf{1}, \mathbf{1})(0,2) \\
& +(\mathbf{1}, \mathbf{1}, \mathbf{1})(0,-2)+(\mathbf{1}, \mathbf{2}, \mathbf{2})(0,0) \\
& \left(\mathrm{SU}(3), \mathrm{SU}(2), \mathrm{SU}(2)^{\prime}\right)\left(\mathrm{U}(1)_{\mathrm{a}}, \mathrm{U}(1)_{\mathrm{b}}\right)
\end{aligned}
$$

We take a linear combination of $\mathrm{U}(1)_{\mathrm{a}}$ and $\mathrm{U}(1)_{\mathrm{b}}$, respecting the orthogonality of the two, to obtain U(1) charges listed in Table II, at the row (15a) and the columns "Branch of 10" and "Branch of 16". We define

$$
\begin{aligned}
& Q_{R} \equiv-x Q_{\mathrm{a}}-y Q_{\mathrm{b}}, \\
& Q_{A} \equiv-2 y Q_{\mathrm{a}}+3 x Q_{\mathrm{b}},
\end{aligned}
$$

where $Q_{i} \mathrm{~s}(i \in\{\mathrm{a}, \mathrm{b}, R, A\})$ denote the charges of $\mathrm{U}(1)_{i}$. Embedding $R$ in $\mathrm{SU}(2) \times \mathrm{U}(1)_{R}$, we obtain the decomposition of the adjoint representation,

$$
\begin{aligned}
\mathbf{7 8}= & (\overline{\mathbf{8}}, \mathbf{1}, \mathbf{1})(0,0)+(\overline{\mathbf{1}}, \mathbf{3}, \mathbf{1})(0,0)+(\overline{\mathbf{1}}, \mathbf{1}, \mathbf{3})(0,0) \\
& +(\overline{\mathbf{1}}, \mathbf{1}, \mathbf{1})(0,0)+(\overline{\mathbf{1}}, \mathbf{1}, \mathbf{1})(0,0) \\
& +(\overline{\mathbf{6}}, \mathbf{1}, \mathbf{1})(-2 x,-4 y)+(\overline{\mathbf{6}}, \mathbf{1}, \mathbf{1})(2 x, 4 y) \\
& +(\overline{\mathbf{3}}, \mathbf{1}, \mathbf{2})(-x,-2 y)+(\overline{\mathbf{3}}, \mathbf{1}, \mathbf{2})(x, 2 y) \\
& +(\overline{\mathbf{3}}, \mathbf{2}, \mathbf{1})(-x,-2 y)+(\overline{\mathbf{3}}, \mathbf{2}, \mathbf{1})(x, 2 y) \\
& +(\overline{\mathbf{3}}, \mathbf{1}, \mathbf{1})(-x-y,-2 y+3 x) \\
& +(\overline{\mathbf{3}}, \mathbf{1}, \mathbf{1})(x+y, 2 y-3 x) \\
& +(\overline{\mathbf{3}}, \mathbf{1}, \mathbf{1})(-x+y,-2 y-3 x) \\
& +(\overline{\mathbf{3}}, \mathbf{1}, \mathbf{1})(x-y, 2 y+3 x) \\
& +(\overline{\mathbf{1}}, \mathbf{2}, \mathbf{1})(-y, 3 x)+(\overline{\mathbf{1}}, \mathbf{2}, \mathbf{1})(y,-3 x) \\
& +(\overline{\mathbf{1}}, \mathbf{1}, \mathbf{2})(-y, 3 x)+(\overline{\mathbf{1}}, \mathbf{1}, \mathbf{2})(y,-3 x) \\
& +(\overline{\mathbf{1}}, \mathbf{1}, \mathbf{1})(-2 y, 6 x)+(\overline{\mathbf{1}}, \mathbf{1}, \mathbf{1})(2 y, 6 x) \\
& +(\overline{\mathbf{1}}, \mathbf{2}, \mathbf{2})(0,0) .
\end{aligned}
$$

We find that $y= \pm 2$ provides the SM Higgs doublet by comparing the $\mathrm{U}(1)_{R}$ charges in the decomposition Eq. (38) with those in Table II, Further investigation shows that we can obtain the SM fermions as well by taking $x=1$ and $y=2$. The resulting field contents are summarized in Table VII. We can explicitly obtain appropriate $\mathrm{U}(1)_{Y}$ hypercharges of the SM particles by taking another linear combination of $\mathrm{U}(1)_{R}$ and $\mathrm{U}(1)_{A}$ as

$$
Q_{Y} \equiv-\frac{6}{11} Q_{R}+\frac{7}{11} Q_{A}
$$




$$
Q_{\alpha} \equiv 19 Q_{R}+2 Q_{A},
$$

where $Q_{Y}$ and $Q_{\alpha}$ are the charges of $\mathrm{U}(1)_{Y}$ and $\mathrm{U}(1)_{\alpha}$, respectively. We thereby obtain SM Higgs, SM fermions and other fermions listed as in Table VIII.

(b) $S / R(9)=\mathrm{G}_{2} \times \mathrm{SU}(3) / \mathrm{SU}(3) \times[\mathrm{SU}(2) \times \mathrm{U}(1)], G=\mathrm{Sp}(16)$, and $F=\mathbf{5 4 4}$.

We embed $R$ in subgroup $\mathrm{SU}(3)_{\mathrm{b}} \times \mathrm{SU}(2) \times \mathrm{U}(1)_{R}$ of $\mathrm{Sp}(16)$ according to the decomposition

$$
\begin{aligned}
\mathrm{Sp}(16) & \supset \mathrm{Sp}(6)_{\mathrm{a}} \times \mathrm{Sp}(6)_{\mathrm{b}} \times \mathrm{Sp}(4) \\
& \supset \mathrm{SU}(3)_{\mathrm{a}} \times \mathrm{Sp}(6)_{\mathrm{b}} \times \mathrm{Sp}(4) \times \mathrm{U}(1)_{R} \\
& \supset \mathrm{SU}(3)_{\mathrm{a}} \times \mathrm{SU}(3)_{\mathrm{b}} \times \mathrm{Sp}(4) \\
& \times \mathrm{U}(1)_{R} \times \mathrm{U}(1)_{A} \\
& \supset \mathrm{SU}(3)_{\mathrm{a}} \times \mathrm{SU}(3)_{\mathrm{b}} \times \mathrm{SU}(2) \times \mathrm{SU}(2) \\
& \times \mathrm{U}(1)_{R} \times \mathrm{U}(1)_{A} .
\end{aligned}
$$

The resulting field contents are summarized in Table VII. We explicitly obtain appropriate $\mathrm{U}(1)_{Y}$ hypercharges of the SM particles by taking combination of $\mathrm{U}(1)_{R}$ and $\mathrm{U}(1)_{A}$ as

$$
\begin{aligned}
& Q_{Y} \equiv 3 Q_{A}-2 Q_{R}, \\
& Q_{\alpha} \equiv-2 Q_{A}-3 Q_{R},
\end{aligned}
$$

where $Q_{i} \mathrm{~s}(i \in\{R, A, Y, \alpha\})$ denote the charges of $\mathrm{U}(1)_{i}$. We thereby obtain SM Higgs, SM fermions and other fermions listed in Table VIII.

(c) $S / R(15 \mathrm{a})=\mathrm{G}_{2} / \mathrm{SU}(2) \times \mathrm{U}(1), G=\mathrm{SO}(13)$, and $F=\mathbf{7 6 8}$.

We decompose $\mathrm{SO}(13)$ as

$$
\begin{aligned}
\mathrm{SO}(13) & \supset \mathrm{SU}(4) \times \mathrm{SO}(7) \\
& \supset \mathrm{SU}(4) \times \mathrm{SU}(2)^{\prime \prime} \times \mathrm{SU}(2)^{\prime} \times \mathrm{SU}(2) \\
& \supset \mathrm{SU}(3) \times \mathrm{SU}(2) \times \mathrm{SU}(2) \times \mathrm{SU}(2) \times \mathrm{U}(1)_{\mathrm{a}} \\
& \supset \mathrm{SU}(3) \times \mathrm{SU}(2) \times \mathrm{SU}(2) \times \mathrm{U}(1)_{\mathrm{a}} \times \mathrm{U}(1)_{\mathrm{b}},
\end{aligned}
$$

where $\mathrm{SU}(2)^{\prime \prime} \sim \mathrm{SO}(3)$ and $\mathrm{SU}(2)^{\prime} \times \mathrm{SU}(2) \sim \mathrm{SO}(4)$. We obtain $\mathrm{U}(1)$ charges listed in Table $\amalg$ at the row of (15a) and the column of "Branch of 10" and "Branch of 16" by taking a linear combination of $\mathrm{U}(1)_{\mathrm{a}}$ and $\mathrm{U}(1)_{\mathrm{b}}$ as

$$
\begin{aligned}
Q_{R} & \equiv \frac{3}{2} Q_{\mathrm{b}}+\frac{1}{2} Q_{\mathrm{a}} \\
Q_{A} & \equiv \frac{3}{2} Q_{\mathrm{b}}-\frac{3}{2} Q_{\mathrm{a}},
\end{aligned}
$$


where $Q_{i}(i \in\{\mathrm{a}, \mathrm{b}, R, A\})$ denote the charges of $\mathrm{U}(1)_{i}$. Embedding $R$ in $\mathrm{SU}(2) \times \mathrm{U}(1)_{R}$, we obtain the field contents summarized in Table VII. We explicitly obtain appropriate $\mathrm{U}(1)_{Y}$ hypercharges of the SM particles by taking another linear combination $\mathrm{U}(1)_{R}$ and $\mathrm{U}(1)_{A}$,

$$
\begin{aligned}
& Q_{Y} \equiv-2 Q_{R}+Q_{A}, \\
& Q_{\alpha} \equiv 16 Q_{R}+6 Q_{A},
\end{aligned}
$$

where $Q_{Y}$ and $Q_{\alpha}$ are the charges of $U(1)_{Y}$ and $U(1)_{\alpha}$, respectively. We thereby obtain SM Higgs, SM fermions and other fermions listed in Table VIII.

(d) $S / R(14)=\operatorname{Sp}(6) / \operatorname{Sp}(4) \times \mathrm{U}(1), G=\operatorname{Sp}(14)$, and $F=\mathbf{3 5 0}$.

We decompose $\operatorname{Sp}(14)$ as

$$
\begin{aligned}
\mathrm{Sp}(14) & \supset \mathrm{Sp}(10) \times \mathrm{Sp}(4) \\
& \supset \mathrm{Sp}(6) \times \mathrm{Sp}(4)^{\prime} \times \mathrm{Sp}(4) \\
& \supset \mathrm{SU}(3) \times \mathrm{Sp}(4)^{\prime} \times \mathrm{Sp}(4) \times \mathrm{U}(1)_{\mathrm{a}} \\
& \supset \mathrm{SU}(3) \times \mathrm{SU}(2) \times \mathrm{Sp}(4) \times \mathrm{U}(1)_{\mathrm{a}} \times \mathrm{U}(1)_{\mathrm{b}} .
\end{aligned}
$$

We obtain U(1) charges listed in Table II at the row of (14) and the columns of "Branch of 10" and "Branch of 16" by taking a linear combinations of $U(1)_{a}$ and $U(1)_{b}$ as

$$
\begin{aligned}
Q_{R} & \equiv \frac{1}{2}\left(-9 Q_{\mathrm{b}}+2 Q_{\mathrm{a}}\right) \\
Q_{A} & \equiv-Q_{\mathrm{b}}-3 Q_{\mathrm{a}},
\end{aligned}
$$

where $Q_{i}(i \in\{\mathrm{a}, \mathrm{b}, R, A\})$ denote the charges of $\mathrm{U}(1)_{i}$. Embedding $R$ in $\operatorname{Sp}(4) \times$ $\mathrm{U}(1)_{R}$, we obtain the resulting field contents summarized in Table VII. We explicitly obtain appropriate $\mathrm{U}(1)_{Y}$ hypercharges of the SM particles by taking another linear combination of $\mathrm{U}(1)_{R}$ and $\mathrm{U}(1)_{A}$ as

$$
\begin{aligned}
Q_{Y} & \equiv-\frac{2}{29}\left(5 Q_{R}+21 Q_{A}\right), \\
Q_{\alpha} & \equiv-\frac{2}{29}\left(14 Q_{R}-5 Q_{A}\right),
\end{aligned}
$$

where $Q_{Y}$ and $Q_{\alpha}$ are the charges of $\mathrm{U}(1)_{Y}$ and $\mathrm{U}(1)_{\alpha}$. We thereby obtain SM Higgs, SM fermions and other fermions listed in Table VIII.

We find four candidates of $(S / R, G, F)$ which give the SM Higgs doublet and at least one generation of the SM fermions in four dimensions. These models, however, generate numerous undesired fields that does not appear in the particle spectrum of the SM as tabulated in Table VIII. These extra fields need to be eliminated to construct a realistic model based on the candidates we found. 


\section{§4. Summary and discussions}

We analyzed gauge-Higgs unification models in a spacetime of the dimensionality $D=14$ under the scheme of the coset space dimensional reduction and exhastively searched for the phenomenologically acceptable models with the dimension of the fermion representation less than 1024.

We first made a complete list of the fourteen-dimensional models by determining the structure of the coset space $S / R$, the gauge group $G$, and the representations $F$ of $G$ for fermions. We obtained a full list of the possible cosets $S / R$ in Table 1 by requiring $\operatorname{dim} S / R=$ 10 and $\operatorname{rank} S=\operatorname{rank} R$. The gauge groups $G$ are determined to have either complex or pseudoreal representations (see Table III), and to lead to one of the following two types of gauge groups after the dimensional reduction to the four-dimensional spacetime: the GUTlike gauge groups such as $\mathrm{SO}(10)(\times \mathrm{U}(1))$ and $\mathrm{SU}(5)(\times \mathrm{U}(1))$, or the Standard-Model (SM)like group which is $\mathrm{SU}(3) \times \mathrm{SU}(2) \times \mathrm{U}(1)(\times \mathrm{U}(1))$ (see Table IV). The representation $F$ of fermions are determined so that the matter content of the SM emerges after the dimensional reduction.

We then analyzed the particle contents of the four-dimensional theories that are induced from each of the sets $(S / R, G, F)$. We found several interesting models in the GUT-like cases.

Among the interesting GUT-like models is the one with $H=\mathrm{SO}(10)(\times \mathrm{U}(1))$, in which one or more fermions of $\mathbf{1 6}$ representation, along with a number of scalars of $\mathbf{1 0}$ representation, are derived in four-dimensional theory. A scalar of $\mathbf{1 0}$ can be interpreted as the electroweak Higgs particle. Two or more fermions of $\mathbf{1 6}$ in the models can account for the

generations of the fermions known in the particle spectra of the SM. The most interesting model in this point of view is the one for $S / R=\mathrm{SO}(7) \times \mathrm{SU}(3) / \mathrm{SO}(6) \times[\mathrm{SU}(2) \times \mathrm{U}(1)]$, $G=\mathrm{SO}(20), F=\mathbf{5 1 2}$, and $H=\mathrm{SO}(10) \times \mathrm{U}(1)$. Three fermions of $\mathbf{1 6}$ are obtained in this case, suggesting the three generations of the fermions in the SM. The U(1) charges associated to them imply a family symmetry under this suggestion.

Similarly, a number of cases of $H=\mathrm{SU}(5) \times \mathrm{U}(1)$ led to the models that induce fermions of $\overline{\mathbf{5}}$ and $\mathbf{1 0}$ representations with a scalar field of $\mathbf{5}$ representation. Although the three sets of fermions are not obtained in these cases, two of them are obtained for $G=\operatorname{Sp}(14)$, $S / R=\operatorname{Sp}(6) / \operatorname{Sp}(4) \times \mathrm{U}(1)$, and $F=\mathbf{3 5 0}$, and can serve for the understanding of the generations.

We also successfully constructed models for $H=\mathrm{SU}(3) \times \mathrm{SU}(2) \times \mathrm{U}(1) \times \mathrm{U}(1)$, where Higgs particle and a generation of the fermions are found. Many unwanted fermions accompany them, however, and a mechanism to eliminate them is necessary to build a realistic model. 
In contrast, some of the GUT-like cases have only the desired fermions. It is worthwhile to analyze these models in further details. An apparent challenge in the GUT-like cases, however, is the absence of the Higgs particle which breaks the GUT gauge group down to the SM gauge group. We can employ the Hosotani mechanism, also known as the Wilson flux breaking mechanism, to circumvent this difficulty. More detailed analyses are necessary to examine if the models we found interesting work in the phenomenological building of the models.

\section{acknowledgments}

This work was supported in part by the Grant-in-Aid for the Ministry of Education, Culture, Sports, Science, and Technology, Government of Japan (No. 17740131, 18034001 and 19010485) and by MEC and FEDER (EC) Grants No. FPA2005-01678 (T. S.).

\section{References}

1) N.S. Manton, Nucl. Phys. B 158 (1979) 141.

2) D.B. Fairlie, Phys. Lett. B 82 (1979) 97.

3) D.B. Fairlie, J. Phys. G 5 (1979) L55.

4) L.J. Hall, Y. Nomura, and D.R. Smith, Nucl. Phys. B 639 (2002) 307.

5) G. Burdman and Y. Nomura, Nucl. Phys. B 656 (2003) 3.

6) I. Gogoladze, Y. Mimura, and S. Nandi, Phys. Lett. B 562 (2003) 307.

7) C.A. Scrucca, M. Serone, L. Silvestrini, and A. Wulzer, JHEP 02 (2004) 049.

8) N. Haba, Y. Hosotani, Y. Kawamura, and T. Yamashita, Phys. Rev. D 70 (2004) 015010.

9) C. Biggio and M. Quiros, Nucl. Phys. B 703 (2004) 199.

10) K. Hasegawa, C. S. Lim and N. Maru, Phys. Lett. B 604 (2004) 133.

11) N. Haba, S. Matsumoto, N. Okada, and T. Yamashita, JHEP 02 (2006) 073.

12) Y. Hosotani, S. Noda, Y. Sakamura, and S. Shimasaki, Phys. Rev. D 73 (2006) 096006.

13) M. Sakamoto and K. Takenaga, Phys. Rev. D 75 (2007) 045015.

14) C. S. Lim, N. Maru and K. Hasegawa, arXiv:hep-th/0605180.

15) Y. Hosotani and Y. Sakamura, Prog. Theor. Phys. 118 (2007) 935.

16) Y. Sakamura, Phys. Rev. D 76 (2007) 065002.

17) A.D. Medina, N.R. Shah, and C.E.M. Wagner, Phys. Rev. D 76 (2007) 095010.

18) Y. Adachi, C.S. Lim, and N. Maru. Phys. Rev. D 76 (2007) 075009.

19) I. Gogoladze, N. Okada, and Q. Shafi, Phys. Lett. B 659 (2008) 316. 
20) D. Kapetanakis and G. Zoupanos, Phys. Rept. 219 (1992) 1.

21) P. Manousselis and G. Zoupanos, JHEP 11 (2004) 025.

22) A. Chatzistavrakidis, P. Manousselis, N. Prezas, and G. Zoupanos, Phys. Lett. B 656 (2007) 152 .

23) A. Chatzistavrakidis, P. Manousselis, N. Prezas, and G. Zoupanos, Proc. of 3rd Southeastern European Workshop on Challenges Beyond the Standard Model (BW2007), Djerdap, Serbia, 2-9 Sep 2007, arXiv/0712.2717 .

24) F.A. Bais, K.J. Barnes, P. Forgacs and G. Zoupanos, Nucl. Phys. B263 (1986) 557.

25) D. Lüst and G. Zoupanos, Phys. Lett. 165B (1985) 309.

26) K.J. Barnes and M. Surridge, Nucl. Phys. B263 (1986) 557.

27) N.G. Kozimirov and I.I Tkachev, Z. Phys. C36 (1987) 83.

28) K. Plich and A.N. Shellekens, Phys. Lett. B164 (1985) 31.

29) K. Farakos, D. Kapetanakis, G. Koutsoumbas and G. Zoupanos, Phys. Lett. B211 (1988) 322 .

30) D. Kapetanakis and G. Zoupanos, Phys. Lett. B249 (1990) 66.

31) B.E. Hanlon and G.C. Joshi, Phys. Rev. D48 (1993) 2204.

32) A.M. Gavrilik, Heavy Ion Phys.11(2000) 35-41.

33) P. Forgacs and N.S. Manton, Commun. Math. Phys. 72 (1980) 15.

34) E. Witten, Phys. Rev. Lett. 38(1977) 121.

35) R. Jackiw, Acta Phys. Austriaca Suppl. XXII (1980) 383.

36) D. Olive and P. West, Nucl. Phys. B217 (1983) 248.

37) Y.A. Kubyshin, J.M. Mourão and I.P. Volobujev, Nucl. Phys. B322 (1989) 531.

38) Y.A. Kubyshin, and I.P. Volobujev, Theor. Math. Phys. 68 (1986) 225, 368, 885.

39) N.S. Manton, Nucl. Phys. B 193 (1981) 502.

40) G. Chapline and R. Slansky, Nucl. Phys. B 209 (1982) 461.

41) K.J. Barnes, R.C. King and M. Surridge, Nucl. Phys. B 281 (1987) 253.

42) S. Randjbar-Daemi, A. Salam and J. Strathdee, Phys. Lett. 132B (1982) 56.

43) C. Wetterich, Nucl. Phys. B222 (1983) 20, B223 (1983) 109, B244 (1984) 355.

44) R. Bott, Differential and combinatorial topology, (Princeton University Press, Princeton, 1965).

45) W. Mckay and J. Patera, Tables of Dimensions, Indices and branching Rules for Representations of simple Algebra, (Dekker, New York, 1981).

46) R. Slansky, Phys. Rept. 79 (1981) 1.

47) Y. Hosotani, Phys. Lett. B 126 (1983) 309.

48) Y. Hosotani, Phys. Lett. B 129 (1983) 193.

49) E. Witten, Nucl. Phys. B258 (1985) 75. 
50) J.D. Breit, B.A. Ovrut and G.C. Segre, Phys. Lett. B158 (1985) 33.

51) B.R. Greene, K. Kirklin and P.J. Miron, Nucl. Phys. B274 (1986) 575.

52) B.R. Greene, K. Kirklin, P.J Miron and G.G. Rpss, Nucl. Phys. B278 (1986) 667.

53) G. Zoupanos, Phys. Lett. B 201 (1983) 301. 